\title{
UNA REVISIÓN DE LA LITERATURA EN ENSEÑANZA Y APRENDIZAJE DE LOS LOGARITMOS (2000-2013): HISTORIA Y EPISTEMOLOGÍA
}

\section{A REVIEW OF THE LITERATURE ON THE TEACHING AND LEARNING OF LOGARITHMS (2000 - 2013): HISTORY AND EPISTEMOLOGY}

\author{
Jeannette Vargas Hernández \\ PhD Educación Matemática \\ Profesora Titular \\ Universidad Colegio Mayor de Cundinamarca

\section{INTRODUCCIÓN} \\ Con los resultados de esta investigación se \\ busca realizar un aporte teórico en el campo \\ de la educación matemática, en particular a \\ los profesores de precálculo, a través de una \\ clasificación y descripción de indagaciones; \\ en la cual se reconozcan características \\ específicas de las funciones logarítmicas. Se \\ realiza un proceso de revisión y caracterización \\ a partir de la noción de conocimiento didáctico \\ del contenido, con marco de referencia en \\ el desarrollo histórico y epistemológico del
}

concepto, desde la delineación presentada por Vargas (2017).

El camino se siguió con la intención de impactar a la formación de profesores, con los análisis concernientes a reportes de investigación, al establecer una alternativa a inquietudes concernientes a identificar ¿Desde el conocimiento del desarrollo histórico de los conceptos logaritmo y función logarítmica, qué aportes se pueden plantear para su conceptualización por parte de los profesores de matemáticas en formación y en ejercicio? 


\section{ANTECEDENTES}

Se focaliza la búsqueda en un acercamiento a investigaciones que hacen referencia a la evolución de la noción de Conocimiento Didáctico del Contenido (CDC) y al interior de esta noción toman importancia las revisiones que han indagado sobre dos aspectos:

Conocimiento de los profesores de matemáticas - conocimiento referido a la disciplina académica; las matemáticas.

Conocimiento de los profesores sobre la enseñanza de las matemáticas.
Para abordar el estado de la investigación, concerniente a lo enunciado en los párrafos anteriores, recurrimos a una investigación de estudios de caso de profesores universitarios de precálculo de Vargas (2017) y a la discusión que presenta Pinto (2010) en la cual se encuentra una revisión de los trabajos de Shulman (1986) y cómo ha evolucionado la noción CDC, desde que lo planteara Lee Shulman hasta el 2010.

En lo relativo al desarrollo histórico epistemológico se hace uso de la síntesis organizada a partir de Vargas (2013). Así:

\begin{tabular}{|c|c|c|}
\hline ETAPAS & CARACTERÍSTICAS DE LA EVOLUCIÓN & CRONOLOGÍA \\
\hline $\begin{array}{l}\text { Antecedentes: } \\
\text { las relaciones } \\
\text { entre las } \\
\text { progresiones } \\
\text { aritméticas y } \\
\text { geométricas. }\end{array}$ & $\begin{array}{l}\text { El germen del concepto de logaritmo en el trabajo relativo a los } \\
\text { números gigantescos. } \\
\text { Se establece que los términos de la progresión geométrica: } \\
1, r, r^{2}, r^{3}, r^{4}, r^{5}, \ldots \text { se corresponden con los términos de la progresión } \\
\text { aritmética formada por los exponentes } 0,1,2,3,4,5, \ldots \\
\text { Uso de diversos tipos de identidades trigonométricas por toda } \\
\text { Europa para simplificar los cálculos astronómicos; las Reglas de } \\
\text { Prosthaphaeresis, permitían convertir el producto de funciones } \\
\text { circulares en una suma o diferencia. }\end{array}$ & $\begin{array}{l}\text { Arquímedes (aprox. 287- } \\
212 \text { a.C.) en su obra } \\
\text { Arenario o Psammites. } \\
\text { Stifel (1487-1567) en la } \\
\text { Arithmética Integra (1544). } \\
\text { Nicolas Chuquet (1445- } \\
1488) \text { en Le Triparty en } \\
\text { la science des nombres } \\
(1484) \text {. } \\
\text { Siglo XVI. }\end{array}$ \\
\hline $\begin{array}{l}\text { Los inicios } \\
\text { del concepto: } \\
\text { una base } \\
\text { aritmética con } \\
\text { un fundamento } \\
\text { geométrico. }\end{array}$ & $\begin{array}{l}\text { Se nomina el nuevo concepto. Utiliza por primera vez la palabra } \\
\text { logaritmo. } \\
\text { Se inicia el desprendimiento de la mirada sobre progresiones } \\
\text { discretas; se elabora un modelo que involucra variabilidad, } \\
\text { covariación y una búsqueda de continuidad, generando una } \\
\text { definición geométrica y la elaboración de tablas de cálculo. } \\
\text { La noción de base no existió en los sistemas de logaritmos, de } \\
\text { Napier y Bürgi, y la igualdad log } 1=0 \text { era inadmisible en el mismo. } \\
\text { Diferencias de los dos acercamientos al concepto de logaritmo: } \\
\text { - Napier eligió al principio log } 10^{7}=0 \text { y construye la progresión } \\
\text { geométrica de razón } 1-10^{-7} ; \text { un número próximo a } 1 \text {. } \\
\text { Bürgi parte de log } 10^{8}=0 \text { y tomó como razón, para su } \\
\text { progresión geométrica, el número } r=\text {. } \\
\text { Así, la relación log } m<\log n \text { si } m>n \text {, es cierta en el sistema } \\
\text { de Napier, mientras en el de Bürgi se verifica log } m>\text { log } n \\
\text { si } m>n \text {. }\end{array}$ & $\begin{array}{l}\text { Napier (1550-1617) llamó } \\
\text { a sus índices de potencias } \\
\text { o exponentes números } \\
\text { artificiales, y más tarde } \\
\text { se decidió por la palabra } \\
\text { compuesta de las dos } \\
\text { palabras griegas logos } \\
\text { (o razón) y arithmos (o } \\
\text { número). Napier (1614) su } \\
\text { obra Mirifici logarithmorum } \\
\text { canonis descriptio. } \\
\text { Jobst Bürgi (1552-1632) } \\
\text { en Arithmetishe und } \\
\text { geometrische Progress- } \\
\text { Tabulen (1620). }\end{array}$ \\
\hline
\end{tabular}




\begin{tabular}{|c|c|c|}
\hline ETAPAS & CARACTERÍSTICAS DE LA EVOLUCIÓN & CRONOLOGÍA \\
\hline $\begin{array}{l}\text { Generalización } \\
\text { del logaritmo } \\
\text { como exponente } \\
\text { de las potencias. }\end{array}$ & 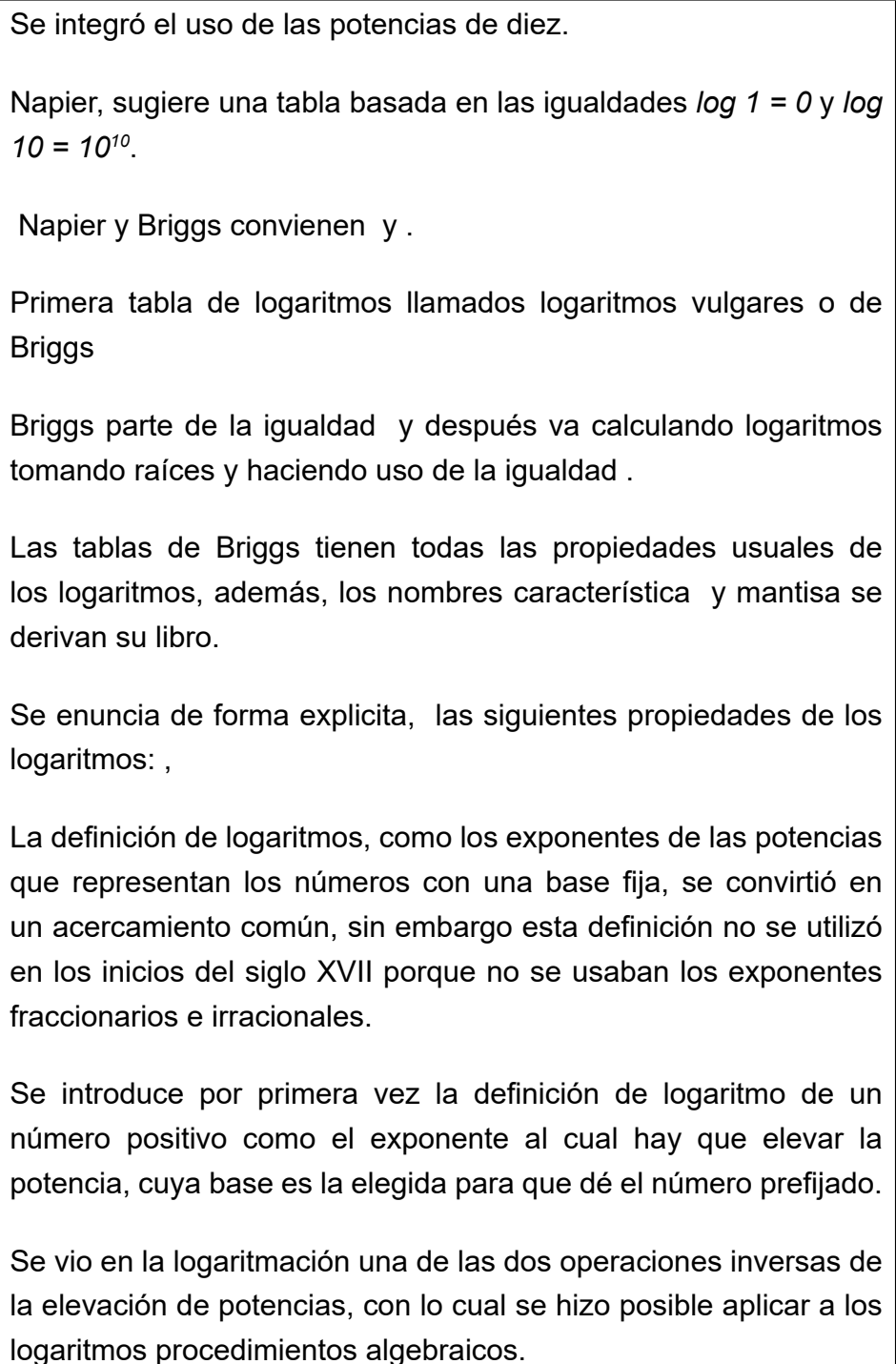 & $\begin{array}{l}\text { Henry Briggs (1561-1639) } \\
\text { Briggs en } 1617 \text { publicó su } \\
\text { obra Logarithmorum chilias } \\
\text { prima } \\
\text { Briggs, en su obra La } \\
\text { Arithmetica Logarítmica } \\
\text { (1624). } \\
\text { William Oughtred (1574 } \\
-1660) \text {. } \\
\text { Euler (1707-1783) define } \\
\text { los logaritmos como } \\
\text { exponentes en 1728. } \\
\text { Euler (1707-1783). }\end{array}$ \\
\hline
\end{tabular}




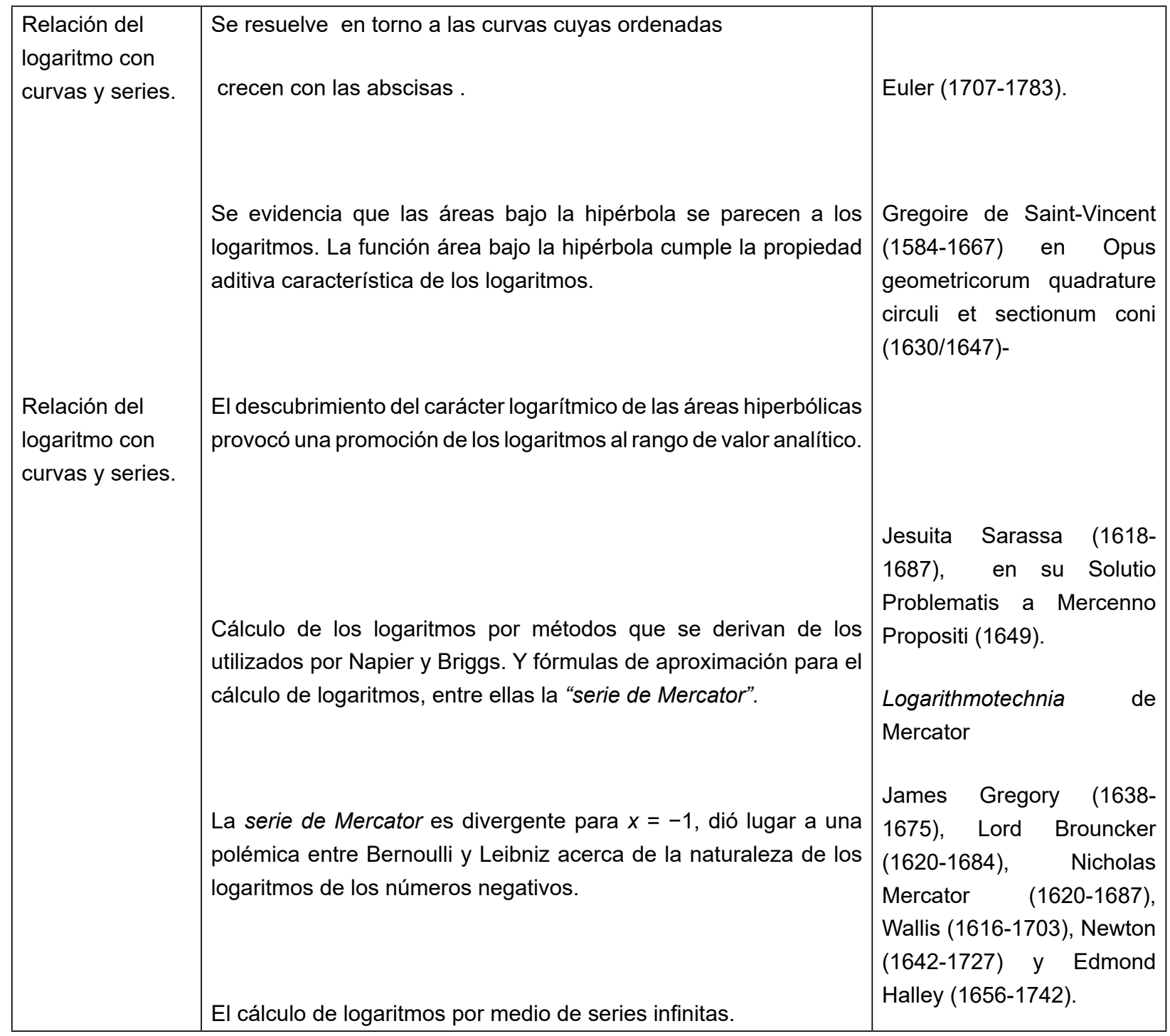




\begin{tabular}{|c|c|c|}
\hline $\begin{array}{l}\text { Logaritmo como } \\
\text { función. }\end{array}$ & $\begin{array}{l}\text { El desarrollo del Análisis Infinitesimal necesitó de una idea de } \\
\text { dependencia funcional, que permitiera aplicar a las distintas } \\
\text { funciones las operaciones del nuevo cálculo, además de la } \\
\text { clasificación de las funciones conocidas. } \\
\text { Se considera que una función es una expresión analítica. } \\
\text { Se define función. } \\
\text { Euler introduce así el logaritmo como función inversa de la } \\
\text { exponencial. Dado un valor afirmativo cualquiera de } y \text { vendrá dado } \\
\text { el valor de } z \text { conveniente para que sea az } y \text {; este valor de } z \\
\text { contemplado en cuanto función de } y \text {, se llama LOGARITMO de } y \text {. } \\
\text { Euler establece que los logaritmos de los números negativos no } \\
\text { infinito de éstos. } \\
\text { También enuncia Euler su regla de oro para los logaritmos, la cual } \\
\text { afirma que si hemos calculado log } y \text { y, entonces se puede calcular } \\
\text { londo b cualquier otra base. }\end{array}$ & $\begin{array}{l}\text { Bernoulli (1667-1748). } \\
\text { Euler (1707-1783) escribió } \\
\text { su Introductio in analysin } \\
\text { infinitorum (1748) que está } \\
\text { dedicada al estudio de las } \\
\text { funciones. }\end{array}$ \\
\hline
\end{tabular}

Tabla A. Evolución de los conceptos logaritmo y funciones logarítmicas.

Las síntesis expuesta en la tabla anterior permiten una mirada general a aspectos del logaritmo y la función logarítmica, sin embargo respecto a los objetivos de nuestra indagación, es importante señalar que en el momento de realizar la revisión en las bases de datos se encontraron, además publicaciones que abordan estudios sobre el desarrollo histórico de dichos conceptos, desde la didáctica matemática. También se ubicaron documentos que contienen interesantes aspectos sobre los conocimientos de los procesos de aprendizaje de los estudiantes y otros que informan sobre la categoría denominada conocimiento de estrategias de enseñanza y representaciones instruccionales.

\section{EL MÉTODO}

Se realiza la indagación, tipo análisis documental, con el fin de hacer uso de la clasificación que se obtenga de los diversos segmentos de los documentos, concerniente al desarrollo histórico de dichos conceptos, de tal forma que dicha clasificación y descripción sea el aporte al conocimiento del profesor.

Se resalta, que en esta indagación, con respecto a algunos de los documentos; tesis doctorales y de maestría, que fueron encontrados en el idioma Inglés, se tomó la decisión de dejar los segmentos en su idioma original, principalmente atendiendo a que una de las exigencias académicas actuales es que los docentes 
tengan el manejo de un segundo idioma que les permita acercarse a la investigación en el idioma original.

\section{FASES DE LA INVESTIGACIÓN}

El desarrollo del presente trabajo se llevó a cabo en tres fases, las cuales no implicaron un orden estricto, pues en algunas ocasiones su desarrollo se dio de manera simultánea o alterna.

Fase 1. Definición y delimitación. Se centra en un estudio teórico que permite definir un marco conceptual; posibilita refinar los objetivos y proporciona claridad sobre el objeto de estudio.

De acuerdo con las diferentes motivaciones e intereses que dieron origen a esta propuesta de investigación, se acude a cuatro tópicos que concretan aspectos de la investigación:

Primero, se señala la fuerza que adquiere la noción Conocimiento Didáctico del Contenido (CDC), para caracterizar las publicaciones y por lo tanto proponerla como un referente en la formación inicial y permanente de los profesores.

Segundo, se ratifica la necesidad de contar con unas categorías de clasificación específicas para la información contenida en los documentos seleccionados.

En tercer lugar, se identifican propuestas relacionadas con aspectos del CDC que no tienen directamente vínculo con el desarrollo histórico del concepto, sin embargo se abordan con la intención de ampliar nuestra mirada y abrir el campo de aporte de esta investigación.

El cuarto aspecto consiste en confirmar como eje transversal los momentos del desarrollo histórico del concepto.

Fase 2. Sobre las categorías de análisis
Con el fin de establecer el marco de referencia bajo el cual se caracterizarían las publicaciones seleccionadas y al compartir la idea de autores como Shulman (1986b y 1987), Smith y Neale (1989), Even (1990) y López (1999); quienes coinciden que el conocimiento de la materia es una condición necesaria aunque, no suficiente para la enseñanza y el aprendizaje del contenido, se tomó la decisión, en esta investigación, de comprender y usar la noción conocimiento didáctico del contenido a través de la discusión que presenta Pinto(2010), en la cual se encuentra una revisión de los trabajos de Shulman (1986b) y estudia cómo ha evolucionado la noción CDC, desde que lo planteara Lee Shulman hasta el 2010.

Dado que existe una diversidad de investigadores que proponen o describen los dominios, componentes, elementos o categorías de conocimiento del CDC, es indispensable seleccionar de ellos las que nos permitan caracterizar las publicaciones de acuerdo con nuestros objetivos de investigación.

Coincidimos con Shulman (1986a) quien estableció y propuso tres componentes esenciales como parte del CDC:

1. El conocimiento del contenido enseñable (más relevante a ser enseñado según el sistema escolar), es decir, el conocimiento de la materia específica para enseñar.

2. Formas de representación (estrategias) para enseñar el área específica.

3. Conocimiento del aprendizaje del estudiante (que incluye las estrategias con las que los profesores ayudan al alumno). 
Al interior de la componente conocimiento del contenido enseñable, nosotros buscamos identificar en los documentos aquellas observaciones y desarrollos que se relacionen con conocimientos de un tópico específico matemático; siendo este uno de los aspectos que nombra Pinto, 2010, quien agrupa los conocimientos del contenido matemático en tres grandes subcomponentes: (a) conocimiento sobre la actividad matemática general, (b) conocimientos por tópico específico matemático y (c) conocimientos sobre el currículo matemático.

De los autores citados por Pinto, 2010 como quienes han orientado su trabajo al estudio del conocimiento del contenido enseñable de forma más exhaustiva, nosotros acudíamos a Even (1990) quien conceptúa el conocimiento del contenido enseñable como conocimiento de la materia del profesor (Teachers' subject matter knowledge) acerca de un tópico específico, consideramos de importancia identificar en los conocimientos del profesor, lo que este autor denomina la fuerza del concepto; entendiendo por ello los aspectos que originan los nuevos conceptos, características únicas del concepto y sus propiedades relevantes, relaciones con otros conceptos.

Lo expuesto en el párrafo anterior lo ubicamos dentro de lo denominado conocimiento de la actividad matemática general y en esta misma subcomponente, teniendo en cuenta nuestro interés en la historia de los conceptos matemáticos, uno de los conocimientos al que pretendemos seguirle la huella es la conocimiento de la historia en cuanto a cambios en las nociones o conceptos, la naturaleza de las explicaciones, de la heurística.

Con el objetivo de explicar que se entiende en esta caracterización como conocimiento de las estrategias de enseñanza y representaciones instruccionales, acudimos a Pinto, J. (2010) quien dado su interés por las representaciones, presenta una recopilación respecto al tipo y diferentes formas de representación. Este autor indica que Shulman (1986a) al interior de esta componente esencial del CDC menciona algunas: analogías, ilustraciones, ejemplos, explicaciones y demostraciones. Llinares, Sánchez y García (1994) optan por adicionar los dibujos y las representaciones gráficas que aparecen en los libros o los realizados por el profesor en el tablero. McDiarmid, Ball yAnderson (1989) incluyen además exposiciones verbales, diagramas, simulaciones, dramatizaciones y análisis de contenido; y destacan las representaciones verbales, simbólicas, gráficas o concretas, como inherentes al contenido matemático. Even y Tirosh (1995) desarrollan ejemplos de representaciones; las preguntas, actividades y discusiones vinculadas con el contenido matemático. Putnam y Borko (2000) nombran otras: modelos y metáforas. Estas representaciones o estrategias instruccionales específicas pueden variar según el contenido o tópico específico que se trate. $(2010, p 26)$.

Es importante anotar que se esperaba encontrar en las publicaciones seleccionadas, una gama importante de representaciones instruccionales, sin embargo previendo que se podían encontrar alusiones a las estrategias usuales con que tradicionalmente se presenta el logaritmo y la función logarítmica, se incluyó la categoría estrategias de enseñanza usuales.

En el estudio, se asumió también que el CDC tiene que ver con la forma como los profesores piensan que hay que ayudar a los estudiantes a comprender un determinado contenido (Marcelo, 2007). En esta línea de ideas, se puede decir que Shulman perfila lo que sería el rol del profesor a partir del dominio del CDC:

El profesor debe de algún modo ser fiel, por un lado, a los posibles significados dentro de la materia y, por otro, a las comprensiones 
que sus estudiantes son capaces de alcanzar. El profesor debe construir puentes entre los significados inherentes al contenido curricular y las construcciones de significados que los estudiantes pueden inventar, idear o discernir (Shulman, 1993, p. 58).

Todo lo anterior se concreta en el esquema 1., que permite esquematizar las categorías de análisis y algunos elementos clave a los que se les siguió el rastro en la caracterización de las publicaciones seleccionadas, a través de la selección de segmentos en estos documentos.
En la investigación en Educación Matemática se ha dado especial atención a la indagación concerniente a la comprensión de los estudiantes de un contenido, la cual ha sido estudiada desde las diversas perspectivas. De esta manera el conocimiento de los procesos de aprendizaje de los estudiantes incluyendo las dificultades de ellos, que los investigadores han reportado, en cada una de las temáticas, pasa a constituir no solo parte del conocimiento de los profesores sino que se incluyó como renglón de la clasificación; se considera que su bosquejo contribuye a dejar impresa la necesidad de incursionar en la lectura de investigaciones alrededor de este tópico.

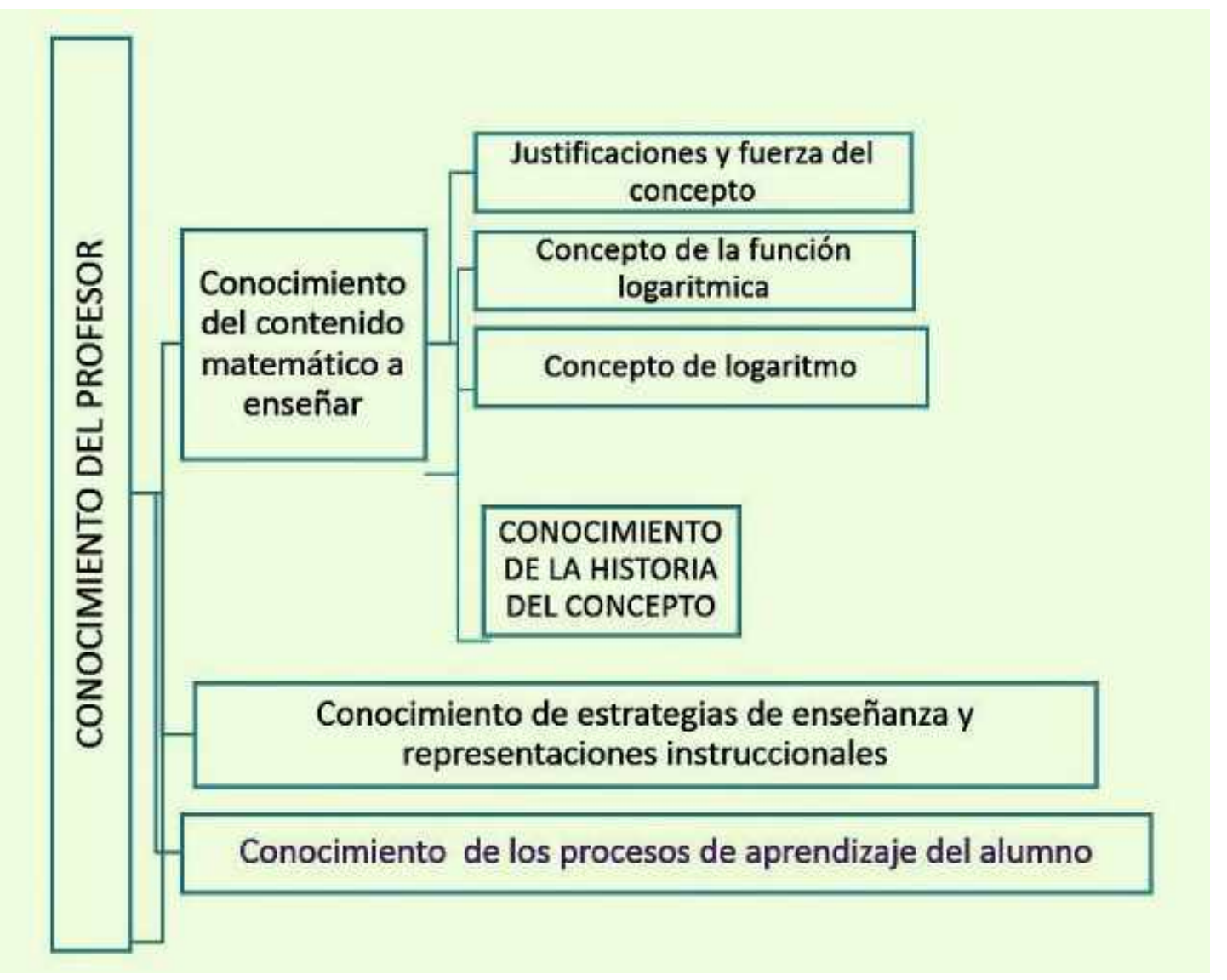

Esquema no. 1 Categorías de análisis; propuesta inicial a partir del CDC 
Fase 3. La documentación concerniente al desarrollo histórico de los conceptos

La búsqueda de los documentos, se realizó indagando en las bases de datos. Luego a través de una lectura general se examinaron aquellos documentos que evidenciaran un análisis, propuesta o contenido respecto al concepto de logaritmo o función logarítmica. Adicional a ello, algunos documentos son seleccionados porque se consideró que se puede encontrar evidencia de la historia del concepto de logaritmo o de la función logarítmica.

\begin{tabular}{|c|c|c|}
\hline Número & & CARACTERÍSTICAS DEL DOCUMENTO \\
\hline \multirow{3}{*}{1} & $\begin{array}{l}\text { TÍTULO } \\
\text { AUTOR - AÑO }\end{array}$ & $\begin{array}{l}\text { Los logaritmos, un abordaje desde la Historia de la Matemática } \\
\text { y las aplicaciones actuales. } \\
\text { Abrate \& Pocholu, } 2007 .\end{array}$ \\
\hline & DOCUMENTO & Artículo \\
\hline & DESCRIPCIÓN & $\begin{array}{l}\text { Se presentan algunas sugerencias de diseño de actividades para } \\
\text { la clase de logaritmos, en el intento de apartarse del tratamiento } \\
\text { tradicional, proponen recuperar la construcción histórica de las } \\
\text { nociones de progresión geométrica y aritmética, y algunas de } \\
\text { sus múltiples aplicaciones. }\end{array}$ \\
\hline
\end{tabular}

Tabla B. Ejemplo de la tabla de clasificación de los documentos seleccionados.

En esta búsqueda no se tuvo ninguna exclusividad en cuanto a su enfoque teórico ni al tratamiento del objeto. La delimitación radica en que fueran documentos enmarcados en la Educación Matemática; para la clasificación se utilizó una tabla como en el ejemplo siguiente (Tabla B).

Identificando título, autor, año, clase de documento y descripción (Tabla B); de esta manera se procedió para los trece documentos resultantes de la búsqueda.

Desde el marco teórico, y como unidades clasificadoras y organizativas de la información, los trece documentos se catalogaron en tres grupos así:

El primer grupo, son los documentos que evidencian una construcción de la representación gráfica de la función logarítmica basadas en elementos de su desarrollo histórico. Estos documentos se examinaron bajo la tutoría que brindó la investigadora principal a dos estudiantes de maestría en docencia de la matemática. Lo anterior como parte del impacto y la función en la formación, de nuevos investigadores, que se pretende cumpla la investigación. ${ }^{1}$

En el segundo grupo, se ubican los documentos que poseen un capítulo o una sección, dedicada exclusivamente a realizar una descripción de los hechos y desarrollo histórico del concepto logaritmo o función logarítmica; dichos documentos son centrales como referentes, puesto que permiten una fundamentación de la evolución del concepto.

Por último, los documentos que quedan por fuera de las dos clasificaciones anteriores, fueron reconocidos como las evidencias, estrategias

1 De aquí en adelante solamente se citaran 9 documentos, dado que las publicaciones del grupo uno fueron las analizadas en la tutoría que brindó la investigadora principal a dos estudiantes de maestría, como parte de la función formadora de investigadores que puede ejercer el grupo BIOMA. 
y apoyo para el profesor de matemáticas para la enseñanza de los logaritmos y función logarítmica. Estos textos, como bien se nombró anteriormente, serán la muestra y sustentación de muchas de las consideraciones a los elementos propuestos para hacer parte del conocimiento didáctico de contenido.

Es de aclarar, que la forma en que se clasificaron los documentos no es excluyente dado que en ellos se deben evidenciar elementos de la clasificación en la que fueron incluidos y se pueden encontrar también ideas de una de las otras clasificaciones.

\section{SÍNTESIS DEL PROCEDIMIENTO}

Se procede con los documentos clasificados en la fase 2, al interior del segundo y tercer grupo; documentos relacionados con resultados de investigación en la enseñanza y aprendizaje de la función logarítmica, así como aquellos que hacen referencia al desarrollo histórico, vinculados con dicho concepto.

Inicialmente se selecciona y lleva a término la lectura de una revisión sobre el desarrollo histórico-epistemológico del logaritmo y la función logarítmica, Vargas, J. (2013) y se contrastan las etapas que allí se describen con los resultados expuestos en Ferrari, M. (2001).

Se examinan y estudian las presentaciones, en cuanto a etapas que describen los cambios "conceptuales" en la evolución del concepto logaritmo y función logarítmica.
A partir de estas lecturas se establece la importancia de rescatar, de la construcción de Jonh Napier (Escoses, 1550, 1617), la indagación y el conocimiento sobre la relación entre la progresión geométrica y la progresión aritmética que está presente en la génesis del logaritmo.

Después de examinar a profundidad la construcción del concepto logaritmo, a través de la revisión que presentan Ferrari (2001); Vargas y González (2007); Vargas (2013), se clasifican los momentos en los documentos, identificando segmentos para cada una de las etapas de la evolución del concepto, referidas en las publicaciones.

La indagación descrita en los anteriores pasos permite establecer el siguiente esquema (Esquema no. 2) que contiene clasificaciones identificadas desde el desarrollo histórico de los conceptos.

Se incorporan estos hallazgos a las categorías de la noción CDC; en cuanto a la revisión del desarrollo histórico, esta evolución del concepto se examina e informa, siguiendo el rastro en cada uno de los segmentos que se clasifican al interior de la categoría Conocimiento de la Historia del contenido y en esta investigación se reconoce también como un elemento intrínseco en la categoría conocimiento de las estrategias de enseñanza y representaciones instruccionales. 


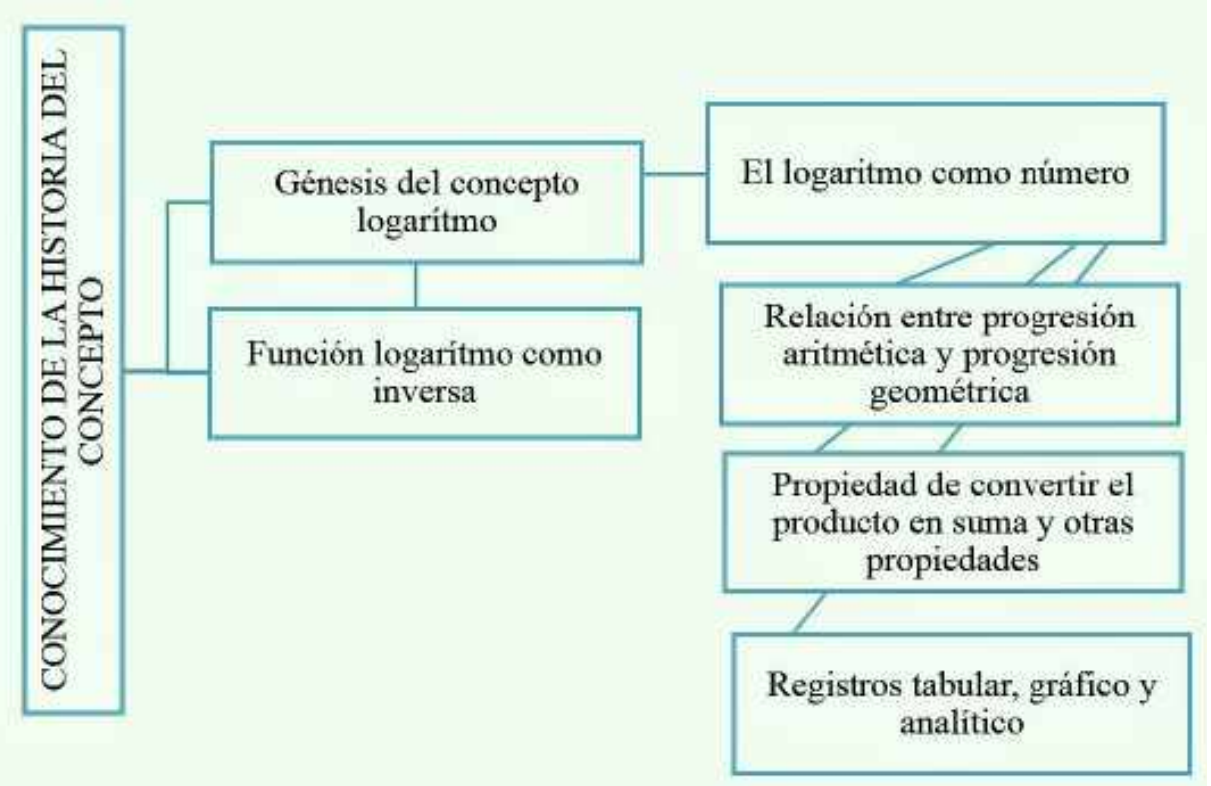

Esquema no. 2. Categorías de análisis de acuerdo con el desarrollo y evolución del concepto

Se desarrolla el análisis documental buscando la triangulación de información, que permitirá establecer consistencia en los cambios "conceptuales" o momentos en la evolución del concepto que se consideran de importancia en la formación de los profesores, con el fin que pasen a integrar el aporte teórico en conexión conocimiento didáctico de contenido.

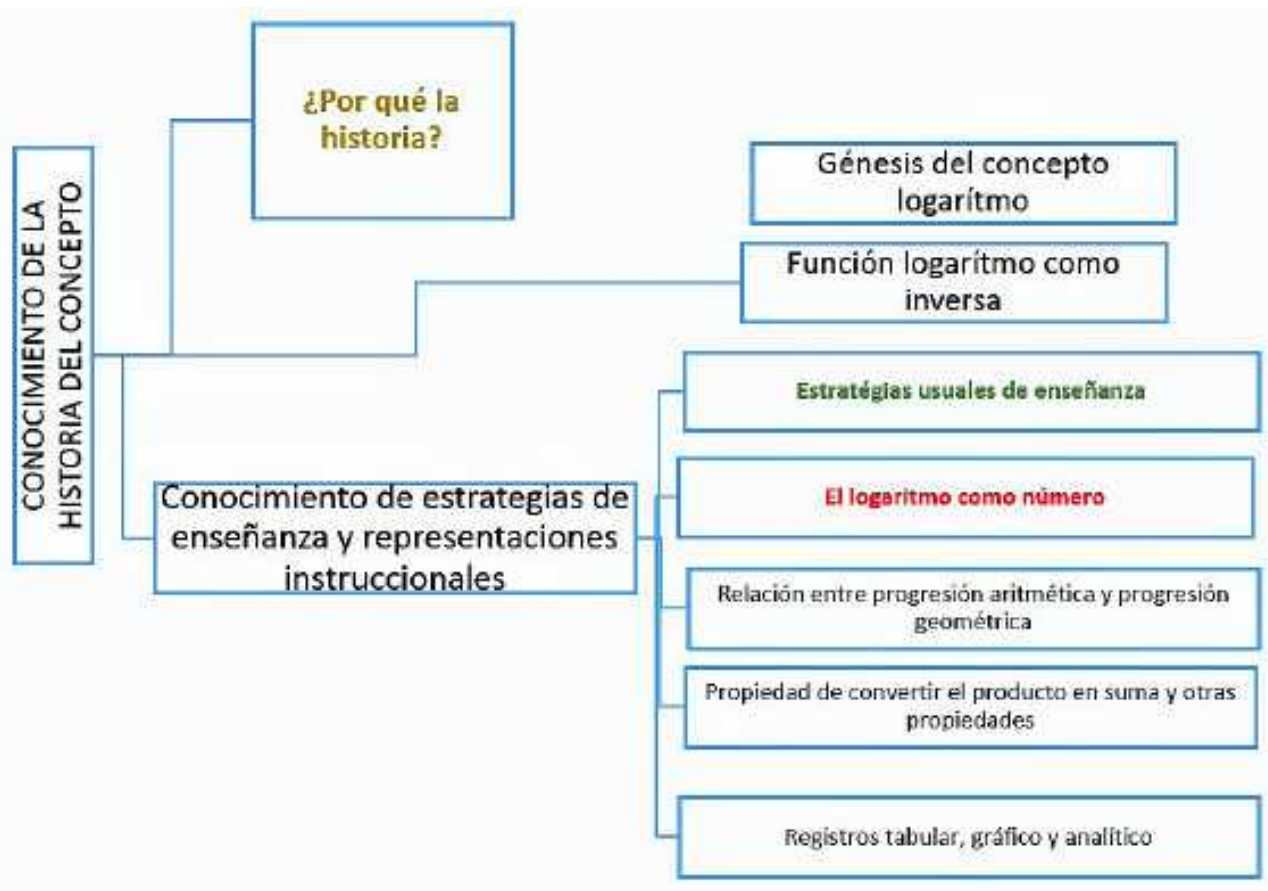

Esquema no. 3. Completando las categorías de análisis 
Dicho análisis documental, en lo relacionado con el desarrollo histórico se realiza sobre ocho publicaciones (Tabla C) que permiten más adelante generar el esquema 4 y adicionar una investigación concerniente a notación de los logaritmos y la comprensión de la función logarítmica. Se tiene así una revisión a nueve documentos.

\begin{tabular}{|c|c|c|c|c|}
\hline Título del documento & Institución & Documento & Autores & Año \\
\hline $\begin{array}{l}\text { 1. Una visión Sociopeistemológica. } \\
\text { Estudio de la Función Logaritmo. }\end{array}$ & $\begin{array}{l}\text { P o l i t e c n i co } \\
\text { Nacional, México } \\
\text { D.F. }\end{array}$ & $\begin{array}{|ll|}\begin{array}{l}\text { Tesis } \\
\text { maestría. }\end{array} & \text { de } \\
\end{array}$ & Ferrari, M. & 2001 \\
\hline $\begin{array}{l}2 . \quad \text { Understanding Mathematical } \\
\text { Concepts: The case of the Logarithmic } \\
\text { Function. }\end{array}$ & Athens, Georgia. & Tesis doctoral & $\begin{array}{l}\text { Kastber, S. } \\
\text { E. }\end{array}$ & 2002 \\
\hline $\begin{array}{l}\text { 3. Elementos historicos para la } \\
\text { enseñanza de la función logaritmica } \\
\text { en la educación básica. }\end{array}$ & \begin{tabular}{|l} 
Universidad del \\
Valle. Colombia.
\end{tabular} & $\begin{array}{|ll|}\text { Tesis } & \text { de } \\
\text { pregrado. } & \end{array}$ & Escobar, $\mathrm{N}$. & 2012 \\
\hline $\begin{array}{l}\text { 4. Segmentos de la historia : la función } \\
\text { logarítmica. }\end{array}$ & $\begin{array}{l}\text { Matemáticas: } \\
\text { E n s e ñ a z a } \\
\text { Universitaria: } \\
\text { Universidad Del } \\
\text { Valle. Colombia. }\end{array}$ & Artículo & $\begin{array}{l}\text { Vargas, J. y } \\
\text { González, } \\
\text { MT. }\end{array}$ & 2007 \\
\hline $\begin{array}{l}\text { 5. Los logaritmos, un abordaje desde } \\
\text { la Historia de la Matemática y las } \\
\text { aplicaciones actuales. }\end{array}$ & \begin{tabular}{|l} 
Experiencias, \\
propuestas y \\
reflexiones para \\
la Clase de \\
Matemáticas. \\
Universidad \\
Nacional de \\
Villamaria, \\
Córdoba. \\
Argentina \\
\end{tabular} & Artículo & $\begin{array}{l}\text { Abrate, R., y } \\
\text { Pocholu, M. }\end{array}$ & 2007 \\
\hline $\begin{array}{l}\text { 6. Análisis de la práctica del docente } \\
\text { universitario de precálculo. Estudio } \\
\text { de casos en la enseñanza de las } \\
\text { funciones exponenciales. }\end{array}$ & $\begin{array}{l}\text { Universidad de } \\
\text { S a I a m a n c a . } \\
\text { España }\end{array}$ & $\begin{array}{l}\text { Tesis doctoral } \\
\text { (Sección 1.3.2) }\end{array}$ & Vargas, J. & 2013 \\
\hline $\begin{array}{l}\text { 7. Algunas consideraciones } \\
\text { didácticas sobre el concepto de } \\
\text { logaritmo y función logarítmica y sus } \\
\text { posibilidades en la educación básica } \\
\text { y media. }\end{array}$ & $\begin{array}{l}\text { Universidad } \\
\text { Nacional de } \\
\text { Colombia. }\end{array}$ & $\begin{array}{|ll|}\text { Tesis } & \text { de } \\
\text { maestría. } & \end{array}$ & $\begin{array}{l}\text { Gacharná, } \\
\text { O. }\end{array}$ & 2012 \\
\hline $\begin{array}{l}\text { 8. An inquiry into high school } \\
\text { students' understanding of logarithms. }\end{array}$ & $\begin{array}{l}\text { Simon Fraser } \\
\text { University, } \\
\text { Burnaby, BC, } \\
\text { Canada. }\end{array}$ & $\begin{array}{|ll|}\text { Tesis } & \text { de } \\
\text { Maestría. } & \end{array}$ & $\begin{array}{l}\text { Berezovsky, } \\
\text { T. }\end{array}$ & 2004 \\
\hline
\end{tabular}




\begin{tabular}{|l|l|l|l|l|}
\hline 9. Students' understanding of & Group for the & Ponencia & Kenney, R. & 2005 \\
logarithmic function notation. & Psychology of & presentada & & \\
& Mathematics & Education, & en la reunión \\
anual del North & & & \\
& Roanoke & $\begin{array}{l}\text { American } \\
\text { Chapter of the } \\
\text { International }\end{array}$ & & \\
& & & \\
\hline
\end{tabular}

Tabla C. Documentos concernientes al desarrollo histórico de logaritmo y función logarítmica.

Reconstruyendo el proceso y estableciendo las piezas con las categorías de análisis, el esquema del estudio fue organizado como se presenta a continuación:

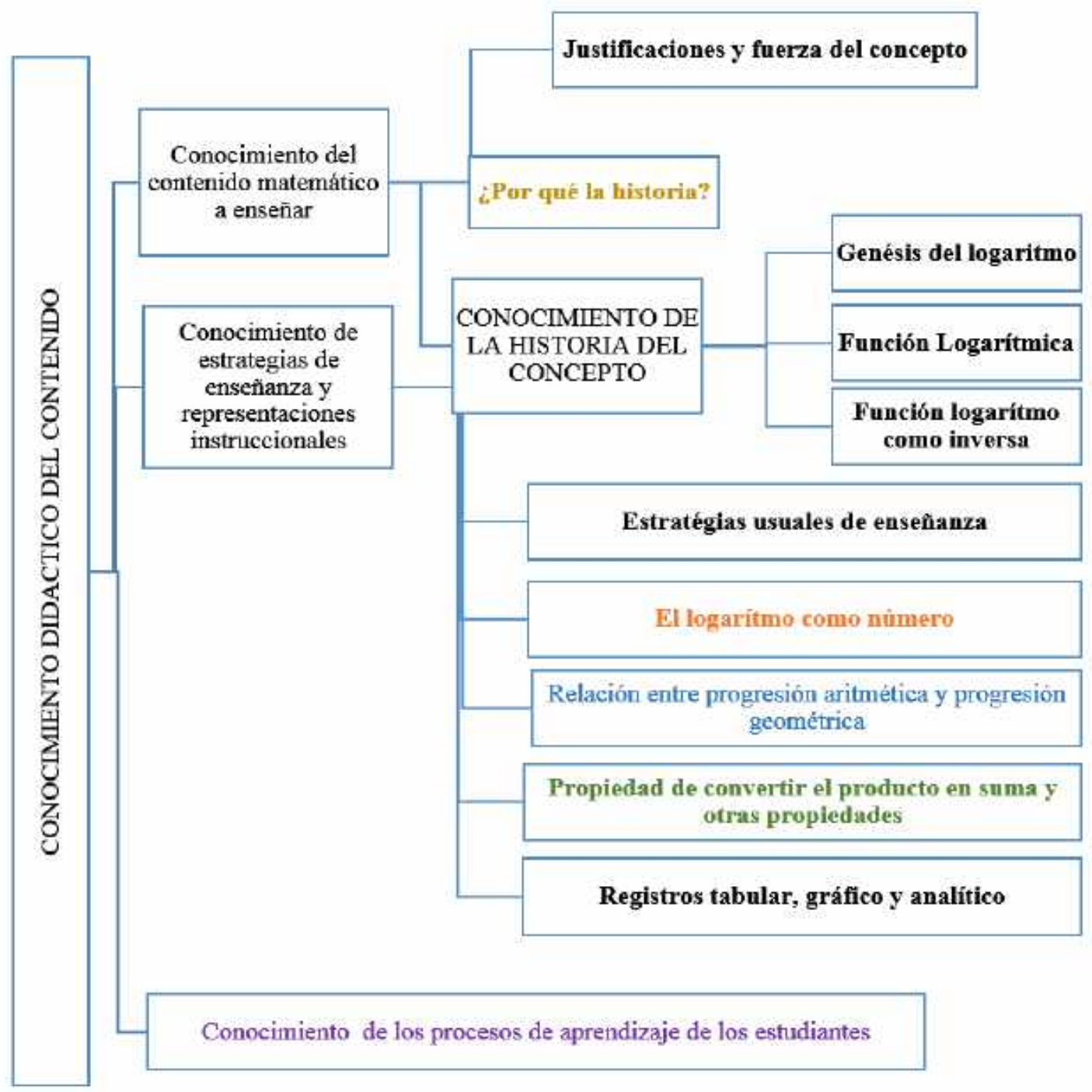

Esquema no. 4. Categorías de análisis para este estudio 
La identificación de segmentos corresponde en general a la categoría Conocimiento Didáctico del Contenido y al interior de ella se establecen las categorías:

- Conocimiento del contenido matemático a enseñar

- Conocimiento de estrategias de enseñanza y representaciones instruccionales

- Conocimiento de los procesos de aprendizaje de los estudiantes.

Estas dos primeras categorías se subdividen con el fin de hacer una descripción más fina de los hallazgos; debido a que ellas corresponden directamente a los objetivos de la investigación.

La categoría Conocimiento de los procesos de aprendizaje del estudiante se integra al estudio, como un aspecto adicional a los objetivos marcados para esta investigación. Se consideró incluir algunos ejemplos de esta categoría dada la importancia que las investigaciones en educación matemática le otorgan ella al interior de la línea de investigación en formación de docentes.

\section{HALLAZGOS}

Se utiliza el orden establecido en el esquema anterior (esquema no. 4) para informar sobre cada una de las categorías. Se recurre a ilustrar con segmentos que describen cada una de ellas y solamente cuando se considera necesario se establecen comentarios adicionales.

Esta sección del documento es la que consideramos de mayor riqueza en el aporte a la formación de los profesores de matemáticas y se complementa con los referentes teóricos del CDC. Todos ellos, junto con los antecedentes, conforman el aporte teórico de nuestro estudio.

\section{JUSTIFICACIÓN Y FUERZA DEL CONCEPTO}

Respecto a esta categoría, se identificaron cuatro publicaciones que exponen argumentos concernientes al efecto de los logaritmos al interior del avance de la matemática, como también a sus usos en la modelación de fenómenos.

En nuestra investigación se considera de interés para la formación de los futuros profesores el conocimiento de cómo ha repercutido la invención del logaritmo en el avance de la matemática y de qué manera son utilizados en las diversas áreas del conocimiento.

Los siguientes segmentos fueron seleccionados porque se considera que hacen referencia a los aspectos que originan los nuevos conceptos, características únicas del concepto y sus relaciones con otros conceptos: 


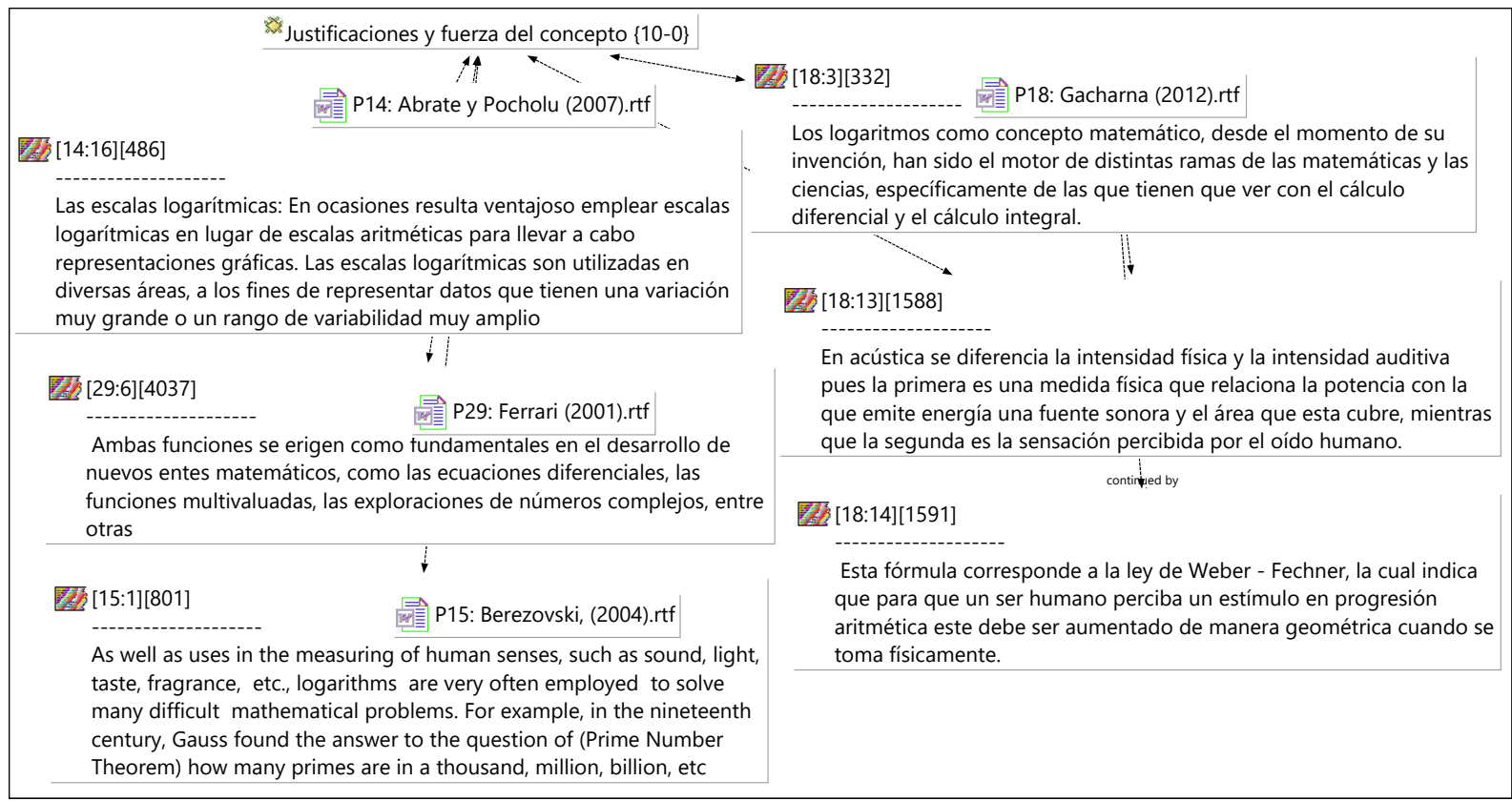

\section{¿POR QUÉ LA HISTORIA?}

Actualmente son privilegiados diferentes caminos para presentar la historia de los conceptos en el aula de clase, en ese caso particular, con el objetivo es intentar dejar de lado la enseñanza enfocada en la aplicación de las propiedades y mostrar los antecedentes analíticos de los logaritmos, al igual que evidenciar el poder de la representación en la creación de conceptos.
Por otro lado está la importancia de conocer métodos y técnicas que desde las ideas iniciales, llevaron concretar un concepto.

A continuación se presenta un ejemplo de los argumentos encontrados en dos de los documentos revisados.

\begin{tabular}{|c|c|c|}
\hline $\begin{array}{l}\text { [27:22][770] } \\
\text { These three important stages in the invention of logarithms provide a }\{9-1\} \\
\text { lens for examining and analyzing students' actions on tasks using } \\
\text { representations of the logarithmic function. In addition, the historical } \\
\text { development of the logarithmic function may a guide for how student } \\
\text { can make sense of both the function and its modern applications. }\end{array}$ & $\begin{array}{l}\text { Th } \\
\text { ch } \\
\text { an } \\
\text { the } \\
\text { rep }\end{array}$ & $\begin{array}{l}\text { [782] } \\
\text { torical development of the logarithmic function discussed in this } \\
\text { r helped me develop tasks to investigate students' understanding } \\
\text { ted as a lens through which to view students' actions. In addition, } \\
\text { torical development highlighted the importance of } \\
\text { entation in the creation of the logarithmic function. }\end{array}$ \\
\hline 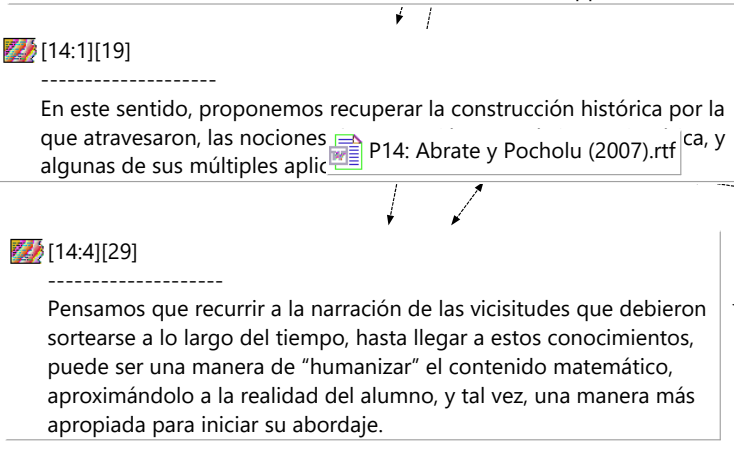 & - explains - & $\begin{array}{l}\text { [14:3][27] } \\
\text { Creemos que muchas veces el modo en que se enseña Matemática } \\
\text { dificulta que se comprenda la relevancia del tema, que se entiendan los } \\
\text { obstáculos del pasado y que adquiera real sentido, al menos en parte, } \\
\text { para muchos de nuestros alumnos. Enseñar contenidos matemáticos } \\
\text { desprovistos de su historia suele acarrear el inconveniente de que } \\
\text { pueden ser concebidos por los alumnos como algo artificioso y } \\
\text { arbitrario de esta ciencia. La perspectiva histórica no sólo permite } \\
\text { conocer cómo se crearon y construyeron los conceptos y las teorías que } \\
\text { hoy manejamos, producto de un trabajo acumulativo, sino también, } \\
\text { faculta para comparar técnicas y métodos actuales con otros que se } \\
\text { utilizaron en el pasado. Así, el quehacer matemático se torna valioso al } \\
\text { poner de manifiesto que un mismo problema se resolvió de maneras } \\
\text { diferentes en distintas épocas. }\end{array}$ \\
\hline
\end{tabular}


Autores como Escobar (2012) presentan en su investigación, las explicaciones de maestros entrevistados, quienes afirman que los retratos, reseñas y anécdotas de los matemáticos pueden también ayudar en la formación de valores humanos y a su vez deben visualizar un sentido epistemológico.

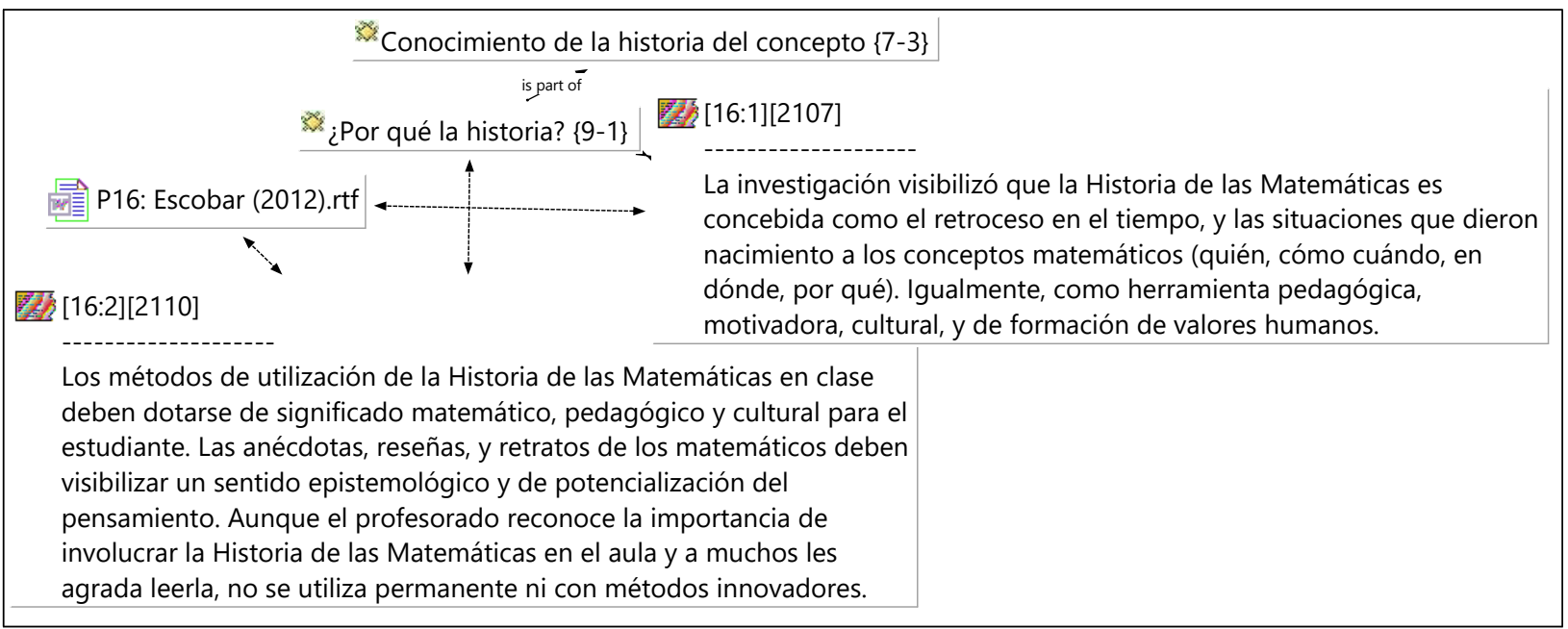

\section{CONOCIMIENTO DE LA HISTORIA DEL CONCEPTO}

El análisis de los documentos permite identificar segmentos, que detallan explicaciones sobre los cambios "conceptuales" o momentos en la evolución del concepto, que se consideran de importancia en la formación de los profesores, con el fin que pasen a integrar su conocimiento didáctica de contenido.

Se presenta a continuación una serie de segmentos extraídos de los documentos, que permiten examinar aspectos del desarrollo del concepto de logaritmo y más delante de la función logarítmica.

\section{GÉNESIS DEL LOGARITMO}

La mayoría de los investigadores abordan el desarrollo histórico de los logaritmos haciendo referencia a Arquímedes (aprox. 287 - 212 AC); Chuquet (1445 - 1488); Stifel (1487 - 1567), deteniéndose a mencionar la identificación de la correspondencia, que se empieza a gestar, entre los términos de una progresión geométrica y los términos de la progresión aritmética.

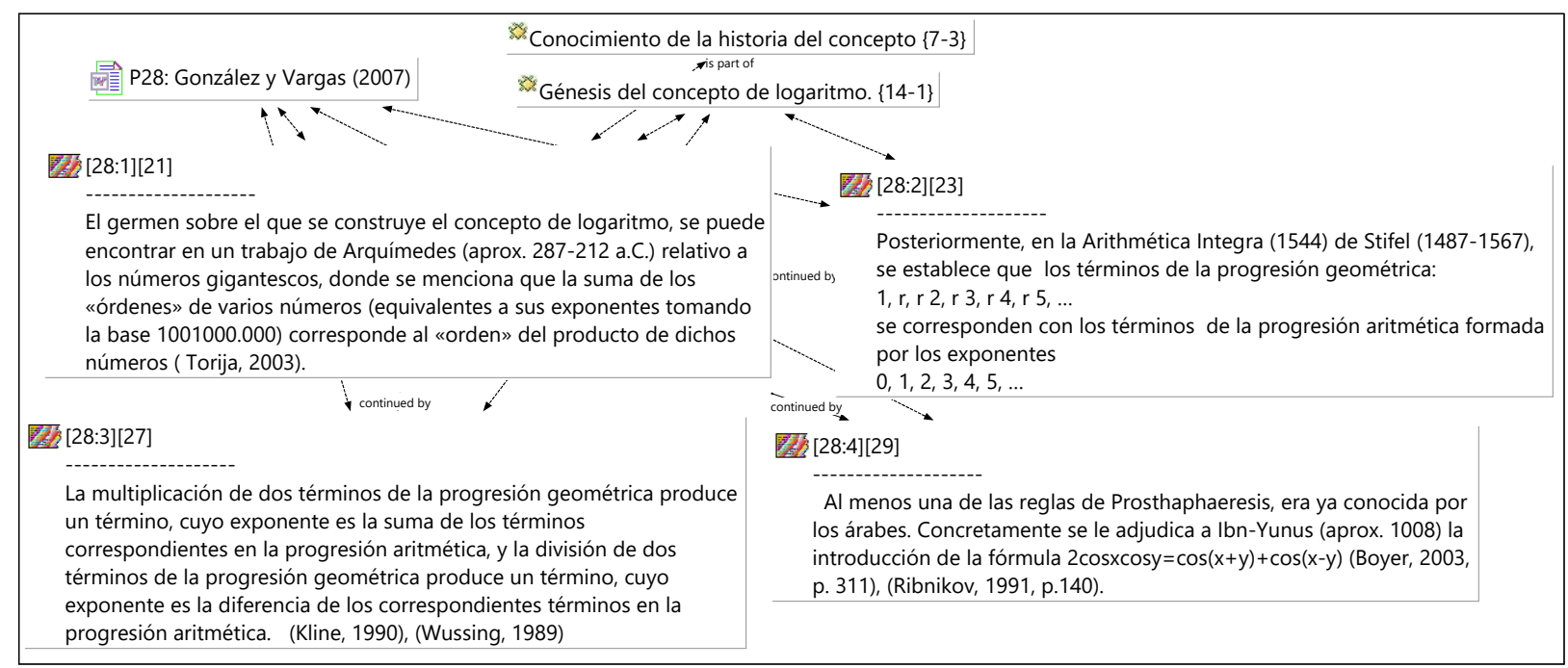


En todos los escritos se encuentra la referencia a Napier con su idea de desarrollar un concepto que permitiera agilizar los cálculos aritméticos de multiplicaciones y divisiones relacionada con las conocidas "Reglas de Prosthaphaeresis". Adicional a ello investigadores como Vargas (2013) y Ferrari (2001) establecen unas etapas del desarrollo del concepto logaritmo; en el primer caso vinculadas con etapas primordialmente cronológicas y epistemológicas y en el segundo caso esencialmente determinadas a partir del contexto en el cual se usaron los logaritmos. En ambos casos resulta de gran riqueza la reseña elaborada, en orden a enriquecer el conocimiento matemático que puedan poseer los profesores de matemáticas.
En el esquema se seleccionaron segmentos que nos permitan mostrarle al profesor, en la construcción diseñada por Napier, que uno de los principales ejercicios analíticos que puede llevar a término un docente es el estudio detallado de la construcción geométrica, junto con las justificaciones y razonamientos alrededor de un movimiento uniforme y un movimiento variable que decrece en proporción a la distancia del punto final.

Se recomienda que al ser usada esta categoría en la formación de profesores, ya sea en una capacitación formal o autoformación se centre la atención en la riqueza que se encuentra al examinar la naturaleza de las explicaciones, de la heurística.

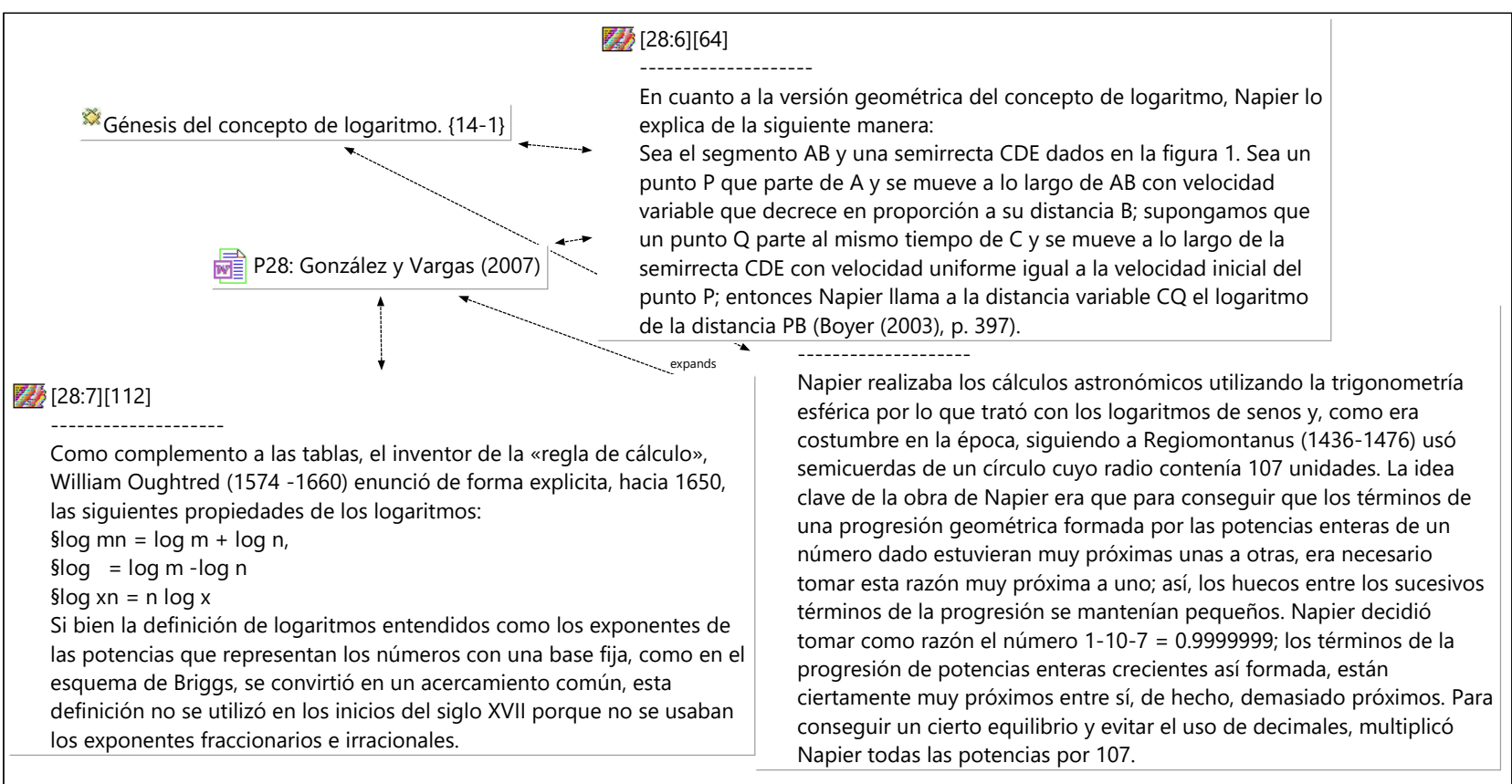

\section{FUNCIÓN LOGARÍTMICA}

Otros aspectos necesarios para focalizar el estudio en la función logarítmica son tanto el paso de lo discreto a lo continuo, como el estudio de las curvas y su posterior cambio de mirada a dichas características a través de expresiones analíticas que denotan las transformaciones, en el concepto de función.
Como lo expresa Ferrari (2001) cuando se logra asociar un número con su logaritmo se hace explicita la función logaritmo. Es de anotar que es Euler quien clasifica dichas funciones como trascendentes. 


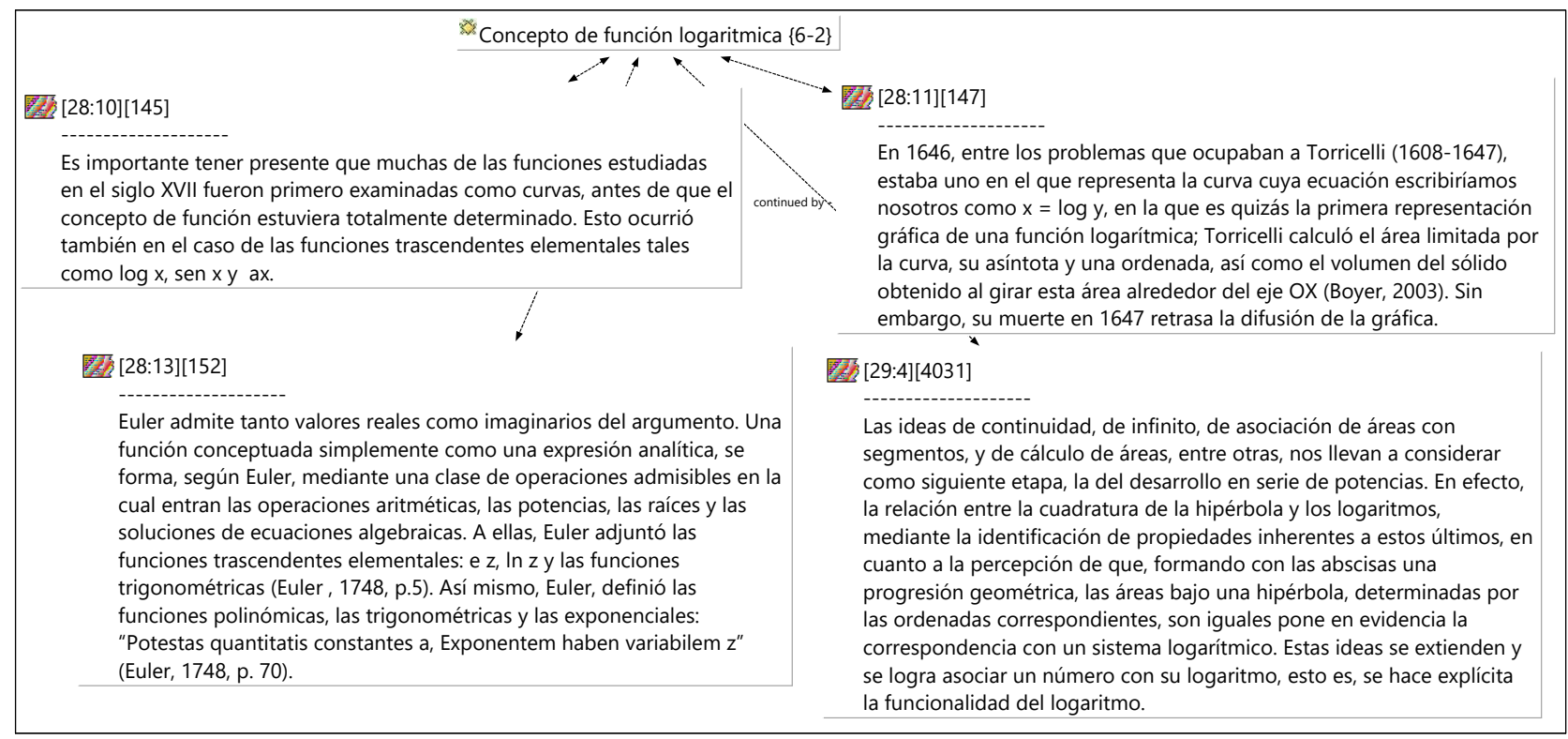

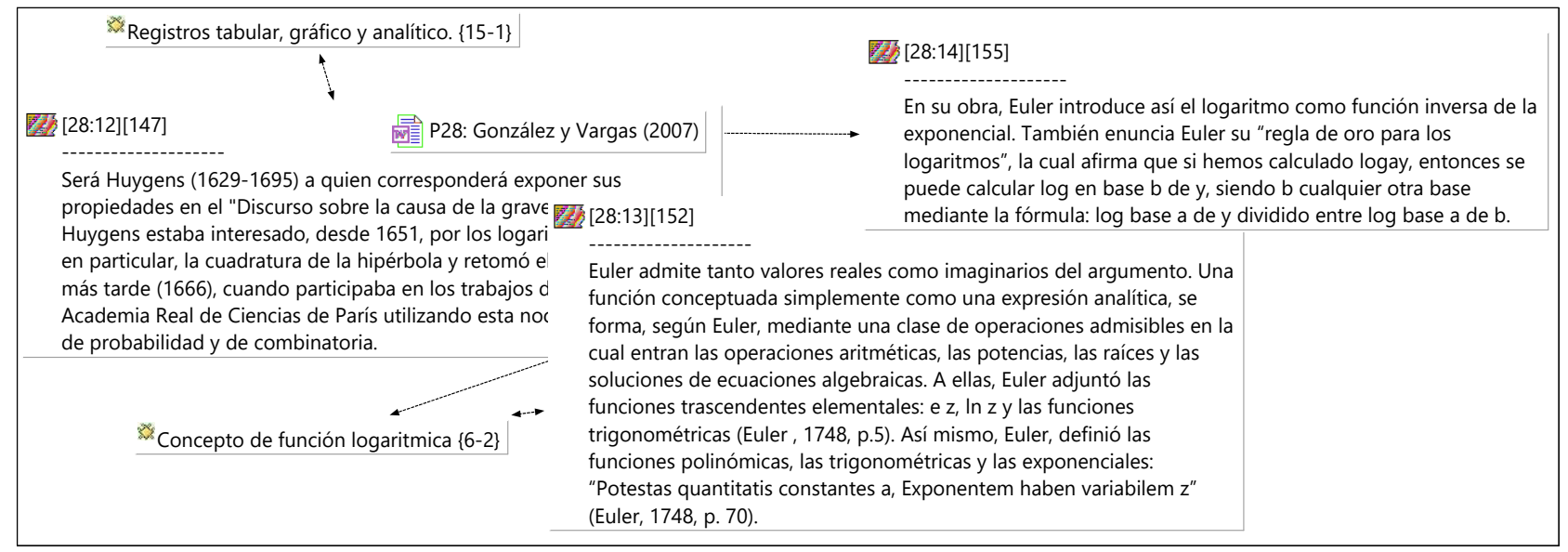

\section{FUNCIÓN LOGARITMO COMO INVERSA}

Se considera de importancia que los profesores de matemáticas incorporen a la presentación tradicional de la función logarítmica como inversa de la función exponencial, sus conocimientos sobre las características de la función logarítmica, además de su caracterización a partir de la progresión geométrica y aritmética y las propiedades de expresadas anteriormente.

La profundidad con que los profesores asocien momentos del desarrollo y su comprensión les permita generar otras estrategias de clase que recaen sobre aspectos como:

Euler fue el primero que vio en la logaritmación una de las dos operaciones inversas de la elevación de potencias, con lo cual se hizo posible aplicar a los logaritmos procedimientos algebraicos (Wieleitner, 1932). Su contribución no se limitó a su definición en términos de exponentes, sino que en 1747 escribió a D'Alembert (1717-1783) explicando el status de los logaritmos de números negativos.

Existe un momento en la evolución de estos conceptos, en el cual se generaliza la idea $y$ define el concepto de logaritmo como exponente, viendo en la logaritmación una de las dos operaciones inversas de la elevación de potencias, con lo cual hace posible aplicar a los logaritmos procedimientos algebraicos. 


\begin{tabular}{|c|c|c|c|}
\hline \multirow{6}{*}{$\begin{array}{l}\text { ED [18:7][752] } \\
\text { El establecimiento de una noción de función por parte de Leonhard } \\
\text { Euler (1707 - 1783) genera que las curvas conocidas adquieran una } \\
\text { correspondiente escritura analítica, entre ellas la curva logarítmica, esto } \\
\text { produjo que se observará la relación entre los exponentes y los } \\
\text { logaritmos, en la observación de las funciones exponencial y } \\
\text { logarítmica, la una como la inversa de la otra. }\end{array}$} & Función logaritmica como inversa. $\{3-1\}$ & {$[29: 5][4037]$} & P29: Ferrari (2001).rtf \\
\hline & Conocimiento de la historia del concepto $\{7-3\}$ & \multirow{3}{*}{\multicolumn{2}{|c|}{$\begin{array}{l}\text { Con Euler quizás se inaugura una nueva etapa, en la cual se vincula } \\
\text { claramente a los logaritmos con la función exponencial, pese a que } \\
\text { encontramos estas ideas en las exploraciones de Leibniz y Bernoulli } \\
\text { acerca de exponenciales. Se los instituye como funciones inversas una } \\
\text { de la otra y se los relaciona con el modelaje de fenómenos de la } \\
\text { naturaleza. }\end{array}$}} \\
\hline & \multirow[b]{2}{*}{ W28:14][155] P28: González y Vargas (2007) } & & \\
\hline & & & \\
\hline & \multirow{2}{*}{\multicolumn{2}{|c|}{$\begin{array}{l}\text { En su obra, Euler introduce así el logaritmo como función inversa de la } \\
\text { exponencial. También enuncia Euler su "regla de oro para los } \\
\text { logaritmos", la cual afirma que si hemos calculado logay, entonces se } \\
\text { puede calcular log en base b de y, siendo b cualquier otra base } \\
\text { mediante la fórmula: log base a de y dividido entre log base a de b. }\end{array}$}} & \\
\hline & & & \\
\hline
\end{tabular}

\section{CONOCIMIENTO DE ESTRATEGIAS DE ENSEÑANZA Y REPRESENTACIONES INSTRUCCIONALES}

\section{ESTRATEGIAS USUALES DE ENSEÑANZA}

De nuestro estudio se desprende que si un profesor de matemáticas desea encontrar la mención sobre estrategias usuales de enseñanza de logaritmo o función logarítmica, puede acudir a los siguientes autores en cuyas publicaciones además se encuentran sugerencia y argumentos sobre formas más elaboradas de conocer el concepto y llevarlo a las aulas.

\section{EL LOGARITMO COMO NÚMERO}

En lo que respecta al logaritmo como número se presentan dos ejemplos que abordan aspectos concernientes a la comprensión de los estudiantes del logaritmo, vinculada con las oportunidades de explicación y acercamientos que se puede hacer en el aula, teniendo presente que cuanto más profundo es el conocimiento del profesor, mayor será la posibilidad de enfocarse en una etapa como en este caso, el logaritmo como número.

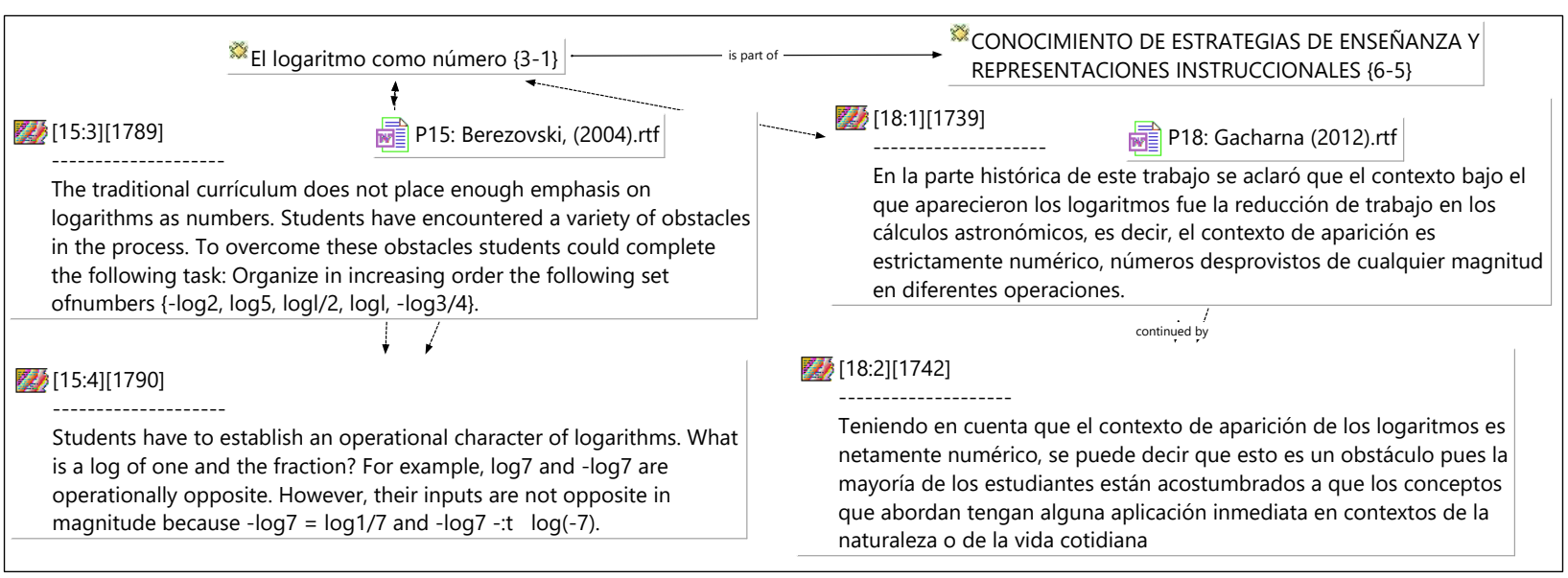

\section{RELACIÓN ENTRE PROGRESIÓN ARITMÉTICA Y PROGRESIÓN GEOMÉTRICA}

La mayoría de los documentos analizados para esta investigación retoman la relación entre la progresión aritmética y la progresión geométrica, no solamente como un episodio del desarrollo histórico sino como la génesis del concepto que puede ser actualmente utilizada en la enseñanza y comprensión del logaritmo y la función logarítmica. A continuación se presenta un ejemplo de los documentos que el profesor puede consultar: 


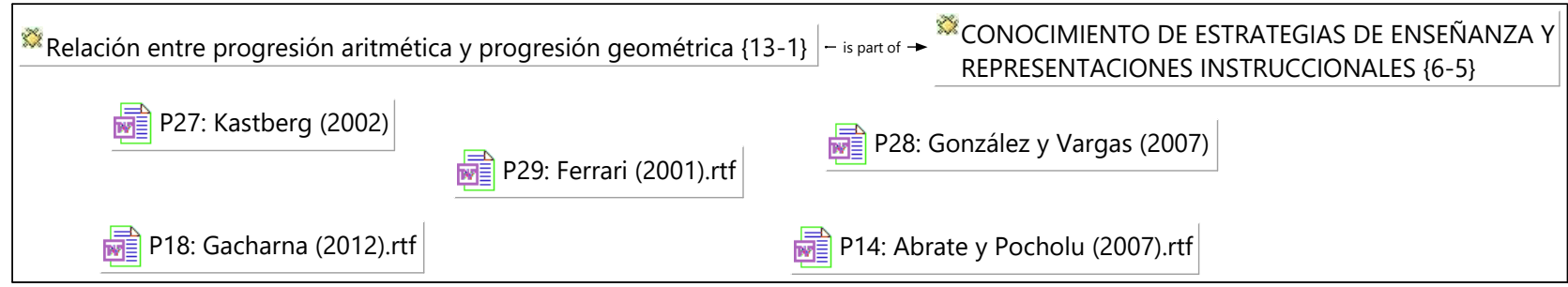

Se enfatiza sobre esta caracterización de la función logarítmica, debido a que de acuerdo con Escobar (2012) en su investigación se establece que los maestros desconocen la razón de la naturaleza de la función logarítmica: la relación entre progresiones geométrica y aritmética. Afirma en su estudio que los logaritmos se estiman como un concepto dependiente de la potenciación, en su aspecto operacional. Propone esta investigadora que se resalte la operatividad individual de ellos, y de hecho se resalte el orden del desarrollo de los logaritmos con respecto a la potenciación, pues es a partir de la logaritmación se establecieron las potencias y es solo hasta Euler (1707-1783) que se hizo una conexión algebraica entre ambas.

\begin{tabular}{|c|c|}
\hline \multirow{2}{*}{\multicolumn{2}{|c|}{$\begin{aligned}- \text { is part of } \longrightarrow \text { CONOCIMIENTO DE ESTRATEGIAS DE ENSEÑANZA Y } \\
\text { REPRESENTACIONES INSTRUCCIONALES }\{6-5\}\end{aligned}$}} \\
\hline & \\
\hline $\begin{array}{l}\text { La primera noción de la relación logarítmica es la comparación entre la } \\
\text { progresión aritmética y la geométrica. Dentro de la profundización de } \\
\text { esta relación se destacan los antecedentes de la progresión geométrica } \\
\text { en la antigüedad, en el renacimiento por Chuquet y Stifel, y la } \\
\text { prostaféresis, aunque no muestra una relación entre las } \\
\text { progresiones aritmética y geométrica si da un acercamiento a la regla } \\
\text { de formación que esta relación implica. }\end{array}$ & $\begin{array}{l}\text { Por otro lado había una curva de la cual no fue tan fácil dar el área bajo } \\
\text { la curva, la llamada hipérbola equilátera se "resistía" a ser cuadrada, } \\
\text { aun cuando la regla de las potencias cuadraba a todas las demás } \\
\text { curvas, hasta que Saint Vincent encontró que la solución tenía que ver } \\
\text { con la relación entre las progresiones aritmética y geométrica, ahora } \\
\text { llamada logarítmica. }\end{array}$ \\
\hline
\end{tabular}

\section{PROPIEDAD DE CONVERTIR EL PRODUCTO EN SUMA Y OTRAS PROPIEDADES}

El poder expresar el producto de un logaritmo como la suma de dos logaritmos o la resta de dos logaritmos a través de un solo logaritmo, caracteriza a la función logarítmica desde su génesis. Esta la intención de buscar un mecanismo matemático que permitiera expresar el producto en suma y las divisiones en restas; explicaciones que se encuentran ampliamente detalladas en los documentos seleccionados en este estudio, alcanza un gran valor en el avance de la comprensión por parte de los profesores, lo cual puede tener importantes repercusiones en las estrategias de enseñanza.

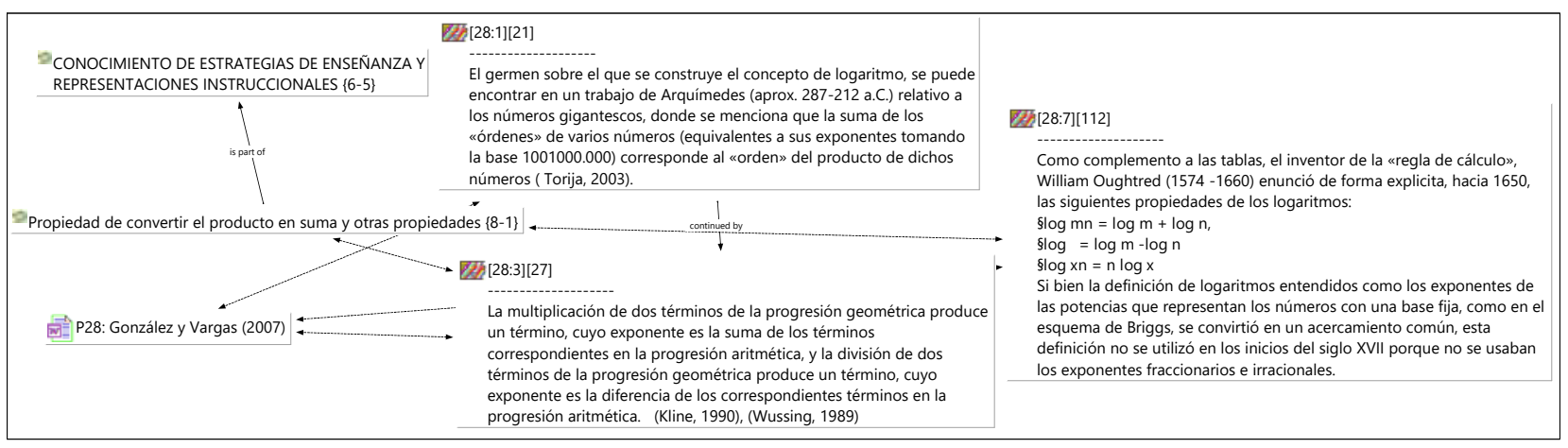


Dada la importancia de lo expresado en el párrafo anterior, se amplió la investigación inicial a otros dos documentos y se expone en la última sección de este informe las conclusiones concernientes a la comprensión de esta cualidad de la función como uno de los aspectos problema en el aprendizaje de ellas. Esta explicación se encuentra en la última sección del informe.

\section{REGISTROS TABULAR, GRÁFICO Y ANALÍTICO}

Los registros de representación son usados en cada una de las etapas de evolución del concepto. Se encuentran en esta red de segmentos una explicación sintetizada y específica sobre elementos matemáticos que fueron la génesis de la función logarítmica y en un primer momento del concepto logaritmo, tales como: progresión aritmética y geométrica, correspondencia entre pares de términos de estas secuencias y la posibilidad de convertir una multiplicación en una adición.

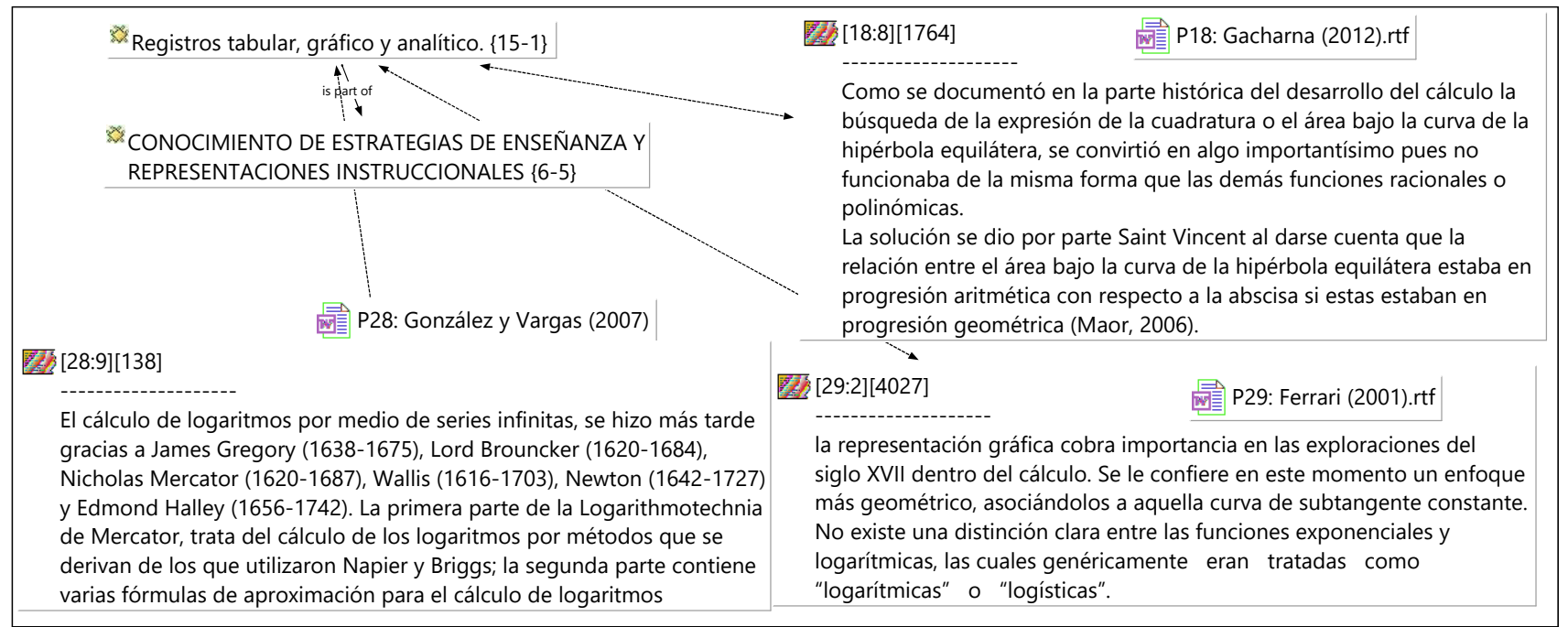

Concerniente a la representación de los logaritmos existe también la necesidad de una comprensión amplia por parte de los profesores, no solo del desarrollo histórico sino también referido a las dificultades de comprensión que su notación conlleva, es por esta razón que en la parte final, sección siguiente, de este trabajo se decidió también ampliar la indagación con un documento adicional.

\section{CONOCIMIENTO DE LOS PROCESOS DE APRENDIZAJE DE LOS ESTUDIANTES}

\section{En el documento titulado "Students'} understanding of logarithmic function notation", la autora afirma que su intención no es buscar específicamente en el proceso de aprendizaje, sino identificar aspectos de la comprensión y concepciones que se han formado en los estudiantes como resultado del aprendizaje. El marco utilizado para este estudio es la teoría de la perspectiva formulada por Gray y Tall (1994), que propone y aquí rescatamos como un aporte a la formación del profesor, lo concerniente a la representación algebraica de la función logarítmica en cuanto a conocer que el uso de símbolos matemáticos permite a los estudiantes considerar conceptos matemáticos para ser a la vez un proceso y un objeto, y que es la capacidad de hacer frente a esta doble naturaleza de la notación que separa al estudiante de matemáticas menos capaz de los más capaces.

En el caso del logaritmo si tomamos la expresión $\log (x)=y$, no es inmediato el análisis que 
permita al investigador decir que representa simultáneamente el proceso de cómo calcular el valor de la función $f(x)$ para un valor particular de $x$ y el objeto, es decir el concepto de función para un valor general de $x$, porque la notación usada lleva a considerar el logaritmo de $\mathrm{x}$, a la vez como un valor de entrada equis pero también como la cantidad de factores que se necesitan, dada la presentación que de ellos se hace en la mayoría de textos: $\log (x)=y$ si $y$ solo si $a^{\wedge} y=x$.

De Kenney (2005), respecto a la categoría que en esta investigación llamamos conocimiento de los procesos de aprendizaje el estudiante, se desea destacar las siguientes afirmaciones:
En la comprensión de la función logarítmica, el profesor, debe asegurarse que el estudiante debe ser capaz de interpretar la notación y símbolos involucrados. En el caso de los libros de textos quienes dan la definición de función logarítmica como ; los estudiantes para ser exitosos en el trabajo con esta función, deben ser capaces de entender esta notación como mostrando tanto el proceso como el objeto (Weber, 2002b). La notación del logaritmo es usada para referirse a una específica función logarítmica y también como un indicador del valor que necesita ser usado en un proceso de exponenciación. El valor de $x$ es ambos, un valor de entrada para la función logarítmica y el producto de y factores de a (Weber, 2002b).

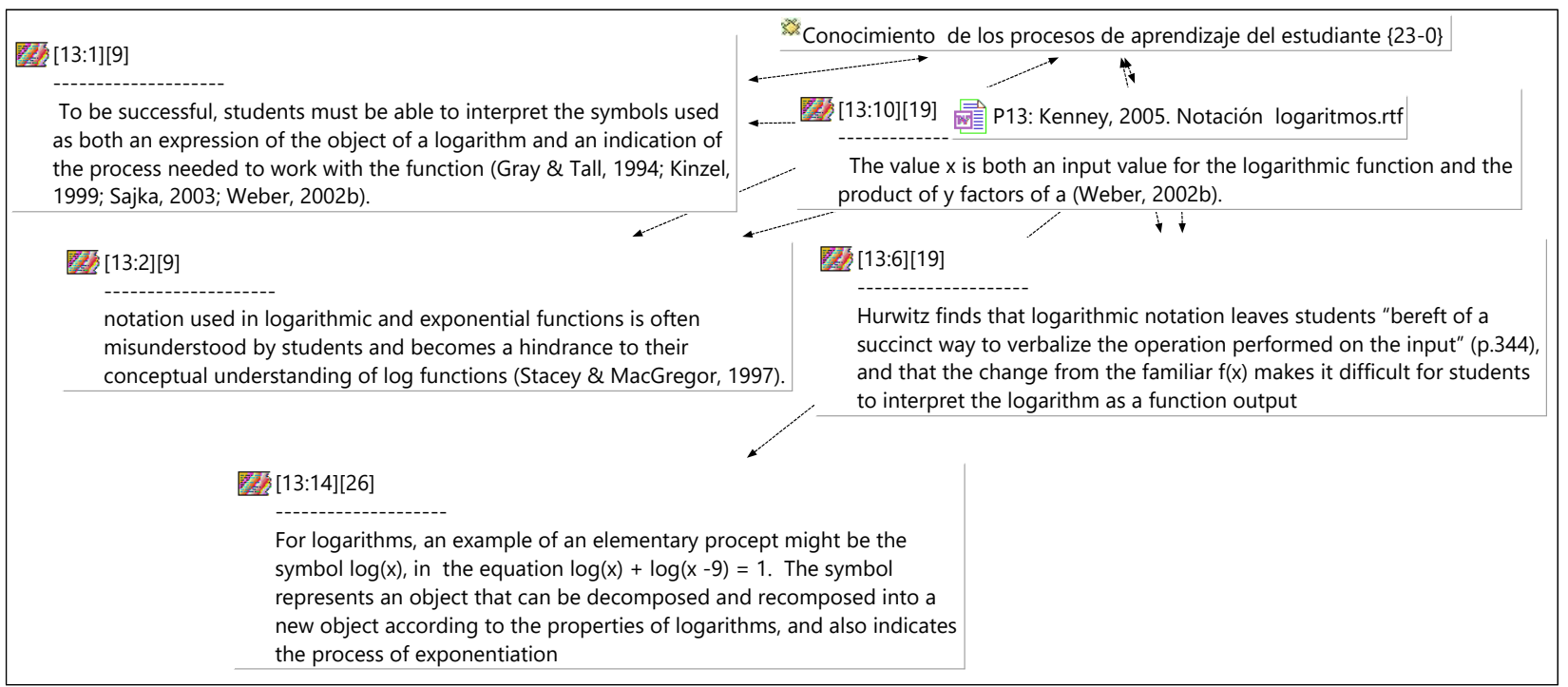

Al respecto Kenney (2005) indaga con varios estudiantes, a través de las siguientes preguntas:

$$
\begin{array}{ll}
\text { a. } & \log _{3}(2)=\text { or } \neq \text { (circle one) } \log _{4}(2) \\
\text { b. } & \log _{3}(1) \quad=\text { or } \neq \text { (circle one) } \log _{3}(1) \\
\text { c. } & \left.\log _{3}(x)+\log _{3}(x+1) \quad=\text { or } \neq \text { (circle one }\right) \quad \log _{5}(x)+\log _{5}(x+1) \\
\text { d. } & \ln (x) \quad=\text { or } \neq \text { (circle one) } \log _{1}(x)
\end{array}
$$

Figure 1. Paired expressions problems from the questionnaire 
Algunos de los hallazgos con los estudiantes son que 26 de los 59 estudiantes utilizaron el argumento para decir que las bases no son iguales, para establecer erróneamente que las expresiones en b no eran iguales. La notación aquí no parecía sugerir un proceso u objeto, tal vez debido a los valores genéricos para las bases. Los estudiantes no vieron relación significativa en los símbolos de registro que apoyaron la actividad matemática (Kinzel, 1999).
Otro resultado sorprendente fue que 35 de 59 estudiantes respondieron a la pregunta $d$ que In (x) era equivalente a $\log (\mathrm{x})$.

La misma falta de comprensión procedimental y conceptual de los logaritmos se exhibió en cuestión c. Aquí, 19 de los 59 estudiantes creían que las expresiones son equivalentes, la mayoría razonó que log era irrelevante, ya que podría ser "anulado". La notación logarítmica, obviamente, no tenía ningún significado para estos estudiantes.

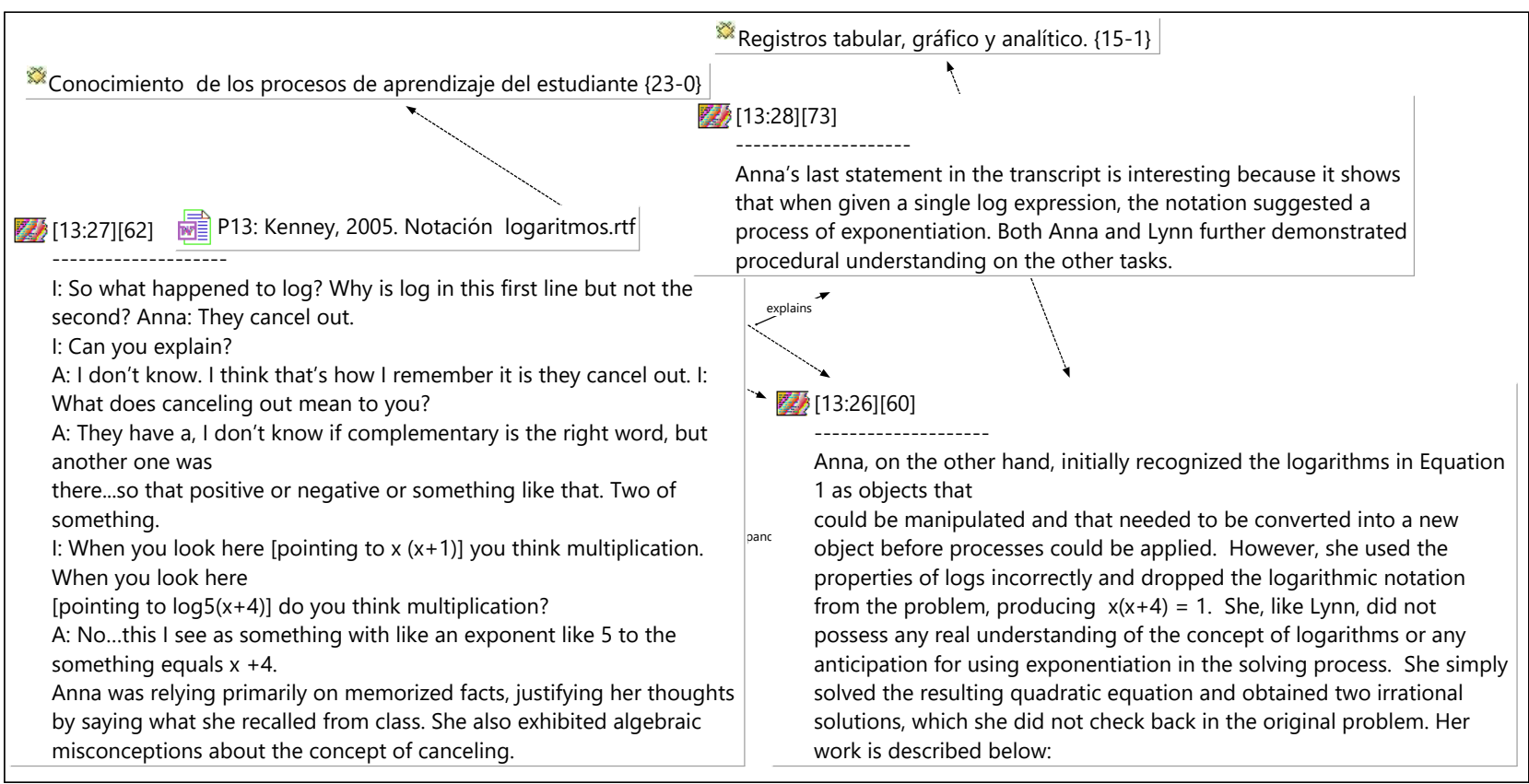

Una de las observaciones interesantes en este procedimiento es que las dos estudiantes recuerdan una propiedad que les permite cancelar y proceden sin darle ningún significado al logaritmo, sin embargo en el caso de Anna en la última frase deja ver que si la expresión de logaritmo es simple como en el caso de , la estudiante expresa que esto será algo como un exponente 5 de algo, igual a . Es decir, en esos casos simples para ella si sugiere un proceso de exponenciación.

Otros hallazgos concernientes a la indagación titulada Students' understanding of logarithmic function notation.

\section{EN CUANTO A LOS CONOCIMIENTOS DEL PROFESOR SOBRE LOS PROCESOS DE APRENDIZAJE DE LOS ESTUDIANTES}

Es importante anotar que la autora de esta publicación concluye que sus descripciones apoyan la idea de Gray y Tall (1994) acerca de que los estudiantes menos capaces no están aprendiendo las técnicas correctas más lentamente, sino que desarrollan sus propias técnicas. De hecho, al final de la entrevista, después de que el investigador ayudó a Lynn a recuperar propiedades de los registros, para que pudiera producir la solución correcta a este problema, ella admitió que ella recordaba 
el hacer el problema de esa manera, pero que su método "parecía mucho más fácil"; ella no hizo ningún reconocimiento de que su método está violando leyes de las matemáticas; los estudiantes examinados en este estudio no mostraron indicios de alejarse de sus diferentes métodos o reconocer sus errores, a pesar de las indicaciones individualizadas que el profesor escribía en la solución que ellos daban a los ejercicios en las evaluaciones.

\section{EN CUANTO CONOCIMIENTO DE ESTRATEGIAS DE ENSEÑANZA Y REPRESENTACIONES INSTRUCCIONALES}

Los estudiantes menos capaces no son simplemente aprendices lentos, pero lo son, de hecho, desarrollando diferentes técnicas para la solución de problemas debido a interpretaciones de los símbolos.

La notación utilizada en funciones logarítmicas y exponenciales es a menudo mal interpretada por los alumnos y se convierte en un obstáculo para su comprensión conceptual de las funciones logarítmicas (Stacey y MacGregor, 1997).

Los estudiantes han aprendido las funciones que han sido presentadas mediante una notación que da claramente una regla acerca de qué hacer con el valor de entrada (Hurwitz, 1999) mientras que la función logarítmica no es considerada de esta misma manera.

En la comprensión de la función logarítmica debe asegurarse de ser capaz de interpretar la notación y símbolos involucrados. En el caso de los libros de textos quienes dan la definición de función logarítmica como ; los estudiantes para ser exitosos en el trabajo con esta función, deben ser capaces de entender esta notación como mostrando tanto el proceso como el objeto (Weber, 2002b). La notación del logaritmo es usada para referirse a una función logarítmica específica y también como un indicador del valor que necesita ser usado en un proceso de exponenciación. El valor de $\mathrm{x}$ es ambos, un valor de entrada para la función logarítmica y el producto de y factores de a (Weber, 2002b).

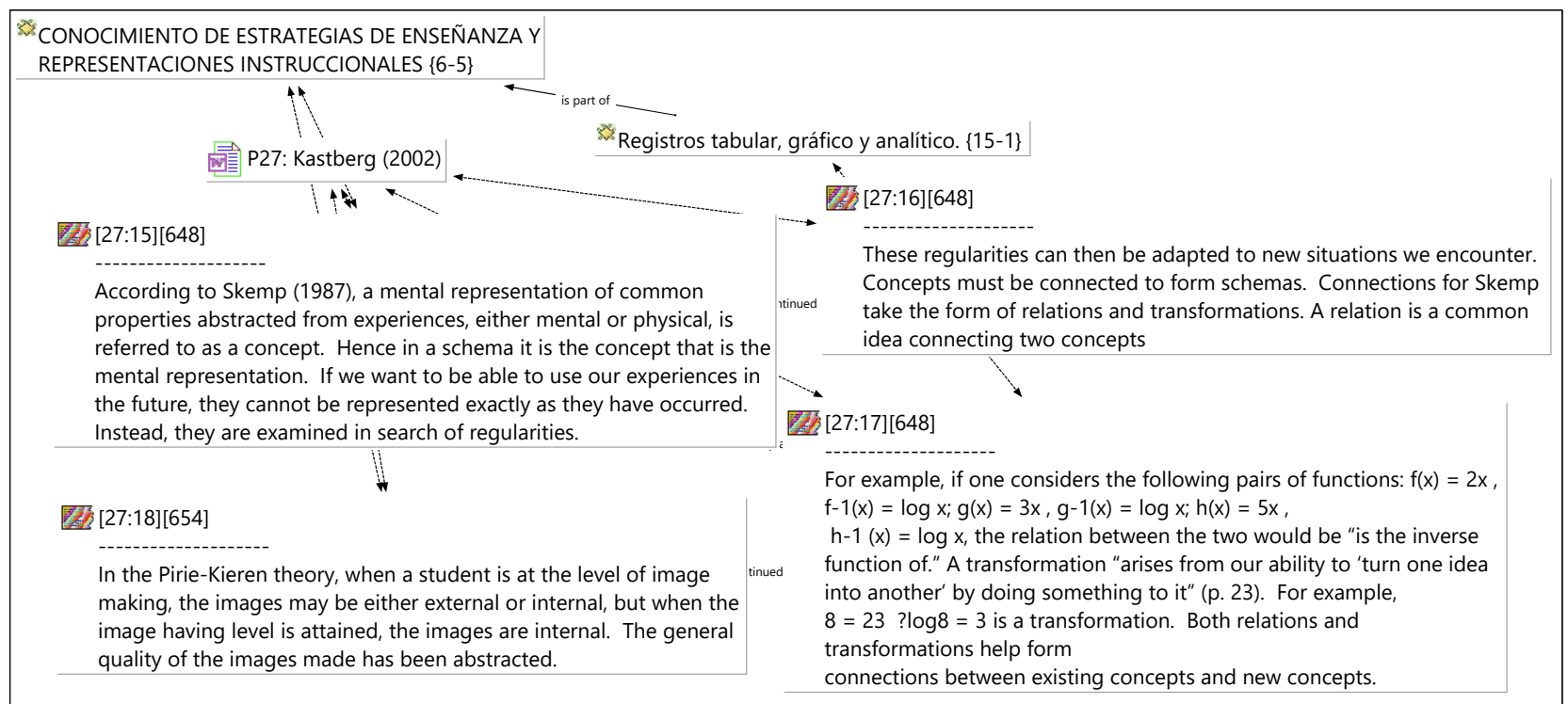


Kastberg (2002) plantea que ella considera que si los estudiantes comprenden la función logarítmica ellos la recordarán. "Si yo enseño diferente el concepto, yo considero que los estudiantes entenderán... Mi investigación produce una colección de material basado sobre aproximaciones que incluyen la introducción tradicional de la función logarítmica como la inversa de la función exponencial. Otras dos aproximaciones que también parecen efectivas: histórica (Toumasis, 1993; Katz 1995) y la función logarítmica como un área (Schoo Mathematics Study Group, 1965)." (Kastberg, p. v).
Kastberg (2002) en lo referido a la función logarítmica, como la inversa de la función exponencial, añade algunas afirmaciones desde otras teorías, que consideramos amplían el campo de comprensión de los profesores en cuanto a la mirada que ellos pueden tener al enseñar dichas funcionesUna transformación "surge de nuestra capacidad de 'convertir una idea en otro' por hacer algo a ella "(Pág. 23). Por ejemplo, $8=2^{3} \rightarrow \log 8=3$ es una transformación. Ambas relaciones y transformaciones ayudan a formar conexiones entre los conceptos existentes y nuevos conceptos.

\section{Conocimiento de los procesos de aprendizaje del estudiante $\{23-0\}$ \\ P27: Kastberg (2002)

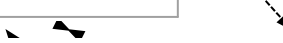

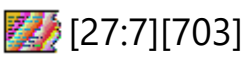 \\ The result of this oversight has been a focus on students' errors as "bugs" (Brown \& Burton, 1978). and the popular term used today misconceptions. Much of what is labeled misconceptions in mathematics could simply be attributable to the reconstructive function of memory. \\ 履 $[27: 8][703]$ \\ One good example of this function of memory are overgeneralizations (Byers \& Erlwanger, 1985) made by students studying the notation of a new concept such as the sum property of logarithms. The students remember this property as $\log b A+\log b B=\log b(A+B)$, an overgeneralization of the distributive law. Much of what students are asked to do in college algebra is based on remembering rather than understanding.}

Concerniente a generalizaciones de propiedades o procedimientos que conllevan a conceptos erróneos de los estudiantes, un ejemplo es la propiedad distributiva que ellos suelen aplicar erróneamente en la suma de logaritmos, de tal manera lo solucionan acudiendo a una sobregeneralización de la ley distributiva, que coinciden con observaciones en la gestión de 
la enseñanza en el aula (Vargas, González y

Vargas, 2020)

Al respecto también se sugiere en esta investigación:

Conocimiento de los procesos de aprendizaje del estudiante $\{23-0\}$
For example, consider the addition of logarithms: log $4+\log 5$. A
student might connect this representation to his or her knowledge of the
distributive property. This connection will result in the following
calculation: log $4+\log 5=\log 9$. Hence the idea is adjoined, but the
connection is not useful. This connection can be modified through a
process that Hiebert and Carpenter call reorganization. Reorganization
can occur when a student reflects on his or her thinking and is aware of
an inconsistency. For example, if a student subsequently sees log $4+$
log $5=\log 20$, he or she may have cause for reorganization. The new
information is not consistent with current mental representations for
adding logarithms.

Como la autora anuncia en la introducción de su investigación, ella estudia dos aproximaciones a la historia del concepto logaritmo y función logarítmica, desde la óptica de su uso en la enseñanza, dado que ella considera que estas parecen efectivas: histórica (Toumasis, 1993; Katz 1995) y la función logarítmica como un área (Schoo Mathematics Study Group, 1965). Encontramos así los siguientes segmentos que hacen referencia a conocimientos que se consideran importantes del dominio del profesor de precálculo y en general los profesores de matemáticas.

En esta misma línea de ideas, la siguiente red de segmentos correspondientes a cada uno de los aspectos que se están caracterizando en nuestra investigación, se encuentran entre cruzados al interior de Conocimiento de estrategias de enseñanza y representaciones instruccionales y Conocimiento de la historia del concepto.

La importancia que tuvo la relación establecida entre una progresión geométrica y una progresión aritmética en la génesis del concepto de logaritmo, es retomada por Kastberg y vinculada a las investigaciones en didáctica de la matemática llevadas a término por Confrey and Smith, quienes en uno de sus estudios examinan la interpretación que los estudiantes pueden hacer, sobre dicha relación, a través de valores dados a ellos en varias tablas de datos; vínculos que también son explícitamente abordados en la construcción de la gráfica de la función logarítmica propuesta por Vargas, Pérez y González (2011).

La literatura citada ayudó a Kastberg a darse cuenta que las representaciones externas y las conexiones que los estudiantes conocen podrían ser usadas como evidencias de sus creencias. Desde allí y con las concepciones de los estudiantes y su aplicación del concepto matemático ella podía hacer sus hipótesis sobre los conocimientos de los estudiantes. 


CONOCIMIENTO DE ESTRATEGIAS DE ENSENANZA Relación entre progresión aritmética y progresión geométrica \{13-1\}
REPRESENTACIONES INSTRUCCIONALES $66-5\}$
Registros tabular, gráfico y analítico. \{15-1\}
unit and a multiplicative rate as primitive actions of students. In
interviews with students, Confrey and Smith presented the students with
a table of values meant to represent the division of a cell over time. The
time, starting at zero, was given in integral values, while the number of
cells was given in powers of 9 . Students identified multiplication by 9 as
the action for moving down the table and division by 9 as the action for
moving up the table. According to Confrey and Smith, the students also
identified various powers of 9 as a constant ratio between terms. For
example, they found the ratio between successive

\section{En las conclusiones presentadas en Kastberg} (2002) se parte de un estudio en el cual los participantes fueron los estudiantes matriculados en álgebra universitaria en un instituto de una comunidad agraria, RC. Todos los instructores tiempo completo de enseñanza de álgebra universitaria en RC durante el otoño, año 2000 fueron contactados y se les pidió si deseaban ser voluntarios para ser observados mientras que realizaban la enseñanza de la función logarítmica - tres visitas- . Se seleccionaron los voluntarios. La selección se basa en la hora y el día que cada instructor estaba enseñando y la fecha en que iba a comenzar la presentación de la función logarítmica.

Para lo anterior, un curso de 3 horas al semestre RC, fue diseñado en torno al concepto de funciones algebraicas y trascendentes; el último tema del semestre en el álgebra universitaria era funciones logarítmicas. Aproximadamente 5 horas de la instrucción se gastaron en este concepto.

Una de las ideas de los estudiantes es que comprender matemáticas implica relacionar 0 reemplazar números en formulas, así presenta el caso de Janine:

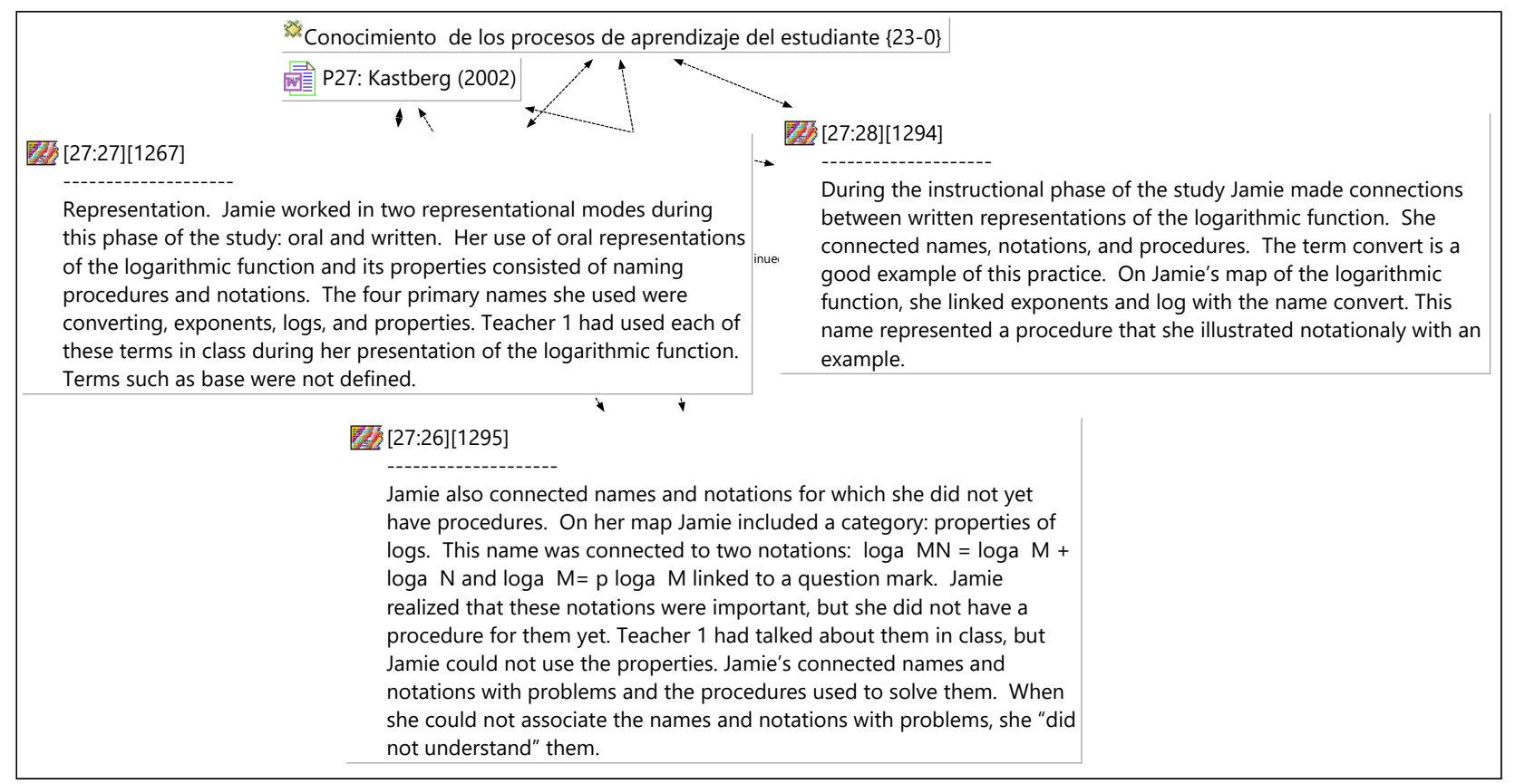


En síntesis en cuanto a las creencias de esta estudiante tenemos que Jamie encontró la función interesante y se ha centrado en convertirse en experta con sus términos, notaciones y procedimientos asociados a la función logarítmica. Esta práctica era compatible con el deseo de Jamie de hacer bien los exámenes. Ella creía que la función logarítmica era como cualquier otro concepto matemático; es decir, si pudiera aprender a asociar tipos de problemas con los nombres, la notación utilizada para resolver el problema y el procedimiento para hacer el problema, la estudiante sentía que en ese caso podría hacer bien en la prueba: ello fue observado como la manifestación de una creencia general

Referente a los aportes en esta investigación:

Se sugiera a los profesores leer el capítulo correspondiente al marco teórico que se utiliza en esta investigación, Understanding mathematical concepts: the case of the logarithmic function, dado que la autora realiza una revisión a la diversidad de conceptualizaciones concernientes a comprensión.

\section{CONOCIMIENTOS DEL PROFESOR SOBRE LOS PROCESOS DE APRENDIZAJE DE LOS ESTUDIANTES}

En el marco teórico de la tesis Understanding mathematical concepts: the case of the logarithmic function la autora analiza que en las cuatro teorías que ella revisa, existen cinco elementos en común, los cuales son: los obstáculos para la comprensión, la modificación para la eficiencia o para superar los obstáculos, base para la comprensión, la representación mental y conexiones. Aunque el lenguaje y las perspectivas de las teorías de los investigadores difieren, cada una de las teorías hace uso de estos cinco elementos.

\section{CONOCIMIENTO DE ESTRATEGIAS DE ENSEÑANZA Y REPRESENTACIONES INSTRUCCIONALES}

Concerniente a generalizaciones de propiedades o procedimientos que conllevan a conceptos erróneos de los estudiantes, un ejemplo es la propiedad distributiva que ellos suelen aplicar erróneamente en la suma de logaritmos, de tal manera lo solucionan acudiendo a una sobregeneralización de la ley distributiva.

Al referirse a la función logarítmica como la inversa de la función exponencial es enriquecedor tener presente que por ejemplo, $8=2^{3} \rightarrow \log 8$ = 3 es una transformación. Consideración que es necesario tener como referente al intentar desentrañar momentos de la comprensión del concepto, dado que una transformación "surge de nuestra capacidad de 'convertir una idea en otra' por hacer algo a ella "(Skemp citado por Kastberg, 1987, 23.).

\section{OTRAS CONCLUSIONES Y RECOMENDACIONES}

Se invita al lector a examinar los esquemas que se presentan, en este informe, en el proceso de identificación de las diferentes categorías de análisis sobre los aspectos concernientes al desarrollo histórico, los cuales le brindan a los profesores en formación y a los docentes en ejercicio un mapa para seguir en cuanto al estudio y comprensión de este tópico específico.

La formación de los docentes en cada concepto y en general en cuanto a los aspectos que concierne a la comprensión; aprendizaje y la enseñanza, es un ambiente vasto con considerables variables, algunas de las cuales se pueden detectar en cada uno de los segmentos que sirven para ilustrar nuestra investigación.

El conocimiento de la historia no per se, sino encaminada y delimitada dentro de algunas de las categorías que hemos utilizado, es una herramienta valiosa, declarada en todas las 
investigaciones consultadas, que se inserta con profundidad en la formación de los profesores.

La riqueza que encierra este documento para la formación de los profesores, se encuentra desde el primer aspecto en el cual se distinguen elementos del CDC y pasa por todas las categorías directamente vinculadas al conocimiento y uso de la historia, para finalmente exhibir su fuerza tanto en los hallazgos en los siete documentos iniciales, como en la investigación se debe continuar; encaminada a las estrategias concernientes a deconstruir el concepto y la trayectoria hipotética de aprendizaje del concepto función logaritmica, en los estudiantes para profesor.

\section{AGRADECIMIENTOS}

Se reconoce el apoyo brindado por la Universidad Colegio Mayor de Cundinamarca mediante el acuerdo 86 de 2018 y el impulso que actualmente recibo para la investigación de la Universidad de Medellín a través del doctor José Alberto Rúa Vásquez con quien estoy desarrollando el diagnóstico para una trayectoria hipotética en el aprendizaje de la función logarítmica.

\section{REFERENCIAS}

Abrate, R. S. y M.D. Pochulu (2007): Los logaritmos, un abordaje desde la Historia de la Matemática y las aplicaciones actuales. Experiencias, propuestas y reflexiones para la clase de matemáticas, Universidd Nacional Villa María, Córdoba, Rep. Argentina.

Boyer, C., Historia de la matemática, Versión de Mariano Martínez Pérez, Alianza Editorial, Madrid, 2003

Berezovski, T. (2004). An inquiry into high school students' understanding of logarithms. Tesis de maestría no publicada, Simon Fraser University, Burnaby, BC, Canada.
Escobar, N. (2012). Elementos historicos para la enseñanza de la función logaritmica en la educación básica. Unibversidad del Valle.

Even, R. (1990). Subject matter knowledge for teaching and the case of functions. Educational Studies in Mathematics, 21(6), 521-544.

Even, R. y Tirosh, D. (1995). Subject-matter knowledge and knowledge about students a sources of teacher presentations of the subject-matter. Educational Studies in Mathematics, 29(1), 1-20.

Ferrari, M. (2001). Una Visión Sociopeistemológica. Estudio de la Función Logaritmo. Centro de Investigación y de Estudios Avanzados del Instituto Politecnico NAcional, México D.F.

Gacharná, O. (2012). Algunas consideraciones didácticas sobre el concepto de logaritmo y de función logarítmica y sus posibilidades en la educación básica y media. Tesis de Maestría. Universidad Nacional de Colombia. See more at: http://www.bdigital.unal.edu. co/11718/\#sthash.WQgMWxdM.dpuf

Giménez, C. A., \& Machın, M. C. (2003). Sobre la investigación en didáctica del análisis matemático. Edición Especial: Educación Matemática, 135.

Gray, E.M., \& Tall, D.O. (1994). Duality, ambiguity, and flexibility: A "proceptual" view of simple arithmetic. Journal for Research in Mathematics Education, 25(2), 116-140.

Kastberg, S. E. (2002). Understanding Mathematical Concepts: The case of the Logarithmic Function. Philosophy. Athens, Georgia. Tesis doctoral. Sin publicar. 
Kenney, R., 2005-10-20 "Students's Understanding of Logarithmic Function Notation" Paper presented at the annual meeting of the North American Chapter of the International Group for the Psychology of Mathematics Education, Hosted by Virginia Tech University Hotel Roanoke \& Conference Center, Roanoke, VA Online<APPLICATION/ PDF>. 2013-12-17 from http://citation. allacademic.com/meta/p24727_index.html

Llinares, S., Sánchez, V. y García, B. M. (1994). Conocimiento del contenido pedagógico del profesor. Tareas y modos de representación de las fracciones. Revista de Educación, 304, 199-225.

López, J. (1999). Conocimiento docente y práctica educativa. El cambio hacia una enseñanza centrada en el aprendizaje. Málaga: Ediciones Aljibe.

McDiarmid, G. W., Ball, D. L. y Anderson, Ch. W. (1989). Why Staying One Chapter Ahead Doesn't Really Work: Subject-Specific Pedagogy. En M. C. Reynolds (Ed.), Knowledge Base for the Beginning Teacher (pp. 193-205). Nueva York: Pergamon, Press.

Pinto, J. (2010). Conocimiento Didáctico del Contenido sobre la representación de datos estadísticos: Estudios de casos con profesores de estadística en carreras de psicología y educación. Universidad de Salamanca.

Putnam, R. \& Borko, H. (2000). El aprendizaje del profesor: implicaciones de las nuevas perspectivas de la cognición. En B. J. Biddle, T. L. Good e I. F. Goodson (Eds.), La enseñanza y los profesores I (pp. 219-309). Barcelona: Paidós

Shulman, L. S. (1986a). Those who understand: Knowledge growth in teaching. Educational Research, 15 (2), 4-14.
Shulman, L. S. (1986b). Paradigms and research programs in the study of teaching. In M.C. Wittrock (Ed.), Handbook of research on teaching. New York: MacMillan.

Shulman, L. S. (1987). Knowledge and teaching: foundations of new reform. Harvard Educational Review, 57 (1), 1-22.

Shulman, L. S. (1993). Renewing the pedadogy of teacher education: the impact of subjectspecific conceptions of teaching. En L. Moreno, y J. M. Vez (Eds.), Las didácticas específicas en la formación de profesores (pp. 53-69). Santiago de Compostela,: Tórculo Edicions

Smith, D. C. y Neale, D. C. (1989). The construction of subject matter knowledge in primary science teaching. Teaching and Teacher Education, 5 (1), 1-20.

Pinto, J.E. (2010). Conocimiento didáctico del contenido sobre la representación de datos estadísticos: estudios de casos con profesores de Estadística en carreras de Psicología y Educación. Tesis doctoral sin publicar. Universidad de Salamanca. España-

Vargas, J. \& González, MT. Segmentos de la historia: la función logarítmica. Matemáticas: Enseñanza Universitaria, XV, (2007). 129144.

Vargas, J., Pérez, M. \& González, M. T. (2011). El logaritmo: ¿cómo animar un punto que relacione una progresión geométrica y una aritmética? En P. P. (Ed.), Memorias del $20^{\circ}$ Encuentro de Geometría y sus Aplicaciones (pp. 129-138). Bogotá, Colombia: Universidad Pedagógica Nacional. 
Vargas, J. (2013/2017). Análisis de la práctica del docente universitario de precálculo. Estudio de casos en la enseñanza de las funciones exponenciales. Ediciones Universidad de Salamanca.

Vargas, J., González, MT., Vargas, N. (2020). Una modelación de mecanismos de construcción y las propiedades de los logaritmos. En Blanco Puentes (Eds.), Resultados del desarrollo de métodos y técnicas de investigación (pp. 251-276). Bogotá, Colombia: Sello editorial Unicolmayor.

Wieleitner, H. (1932). Historia de las matemáticas. Barcelona, España: Editorial Labor. 